\title{
CIRCUMVENTING COMPLEXITY: \\ MOTIF OF \\ INFORMATION \\ AS ANADYOMENE
}


If the history of humankind is viewed from the standpoint of the "obstacle-oriented approach" to complexity, pertaining to localizing and embracing obstructions of any particular type, a naturally arising problematics complicates the border-lines of the most persistent and covariant forms of this stance. The character of temporal persistence and covariance is meant to serve as a criterion for the Platonic "ousia ontos ousa" of history, or "being that truly is". Surprisingly enough, this criterion filters out only two kinds of entities, namely myths and theorems. At a first encounter with this provocative claim, there seems to be a contradiction, but this is only apparent, stemming mainly from a certain type of pre-occupation with "target-oriented" methods instead of "obstacle-oriented ones". Indeed, both myths and theorems become noematic if they are obstacle-embracing, they are just different types of metaphora in relation to communication. Myths and narratives constitute an embodied symbolic process of metaphora around a localized obstacle. Mathematical theorems constitute an abstract symbolic process of metaphora around a localized obstacle. Although "myth" and "math" deceptively seem to occupy opposite sides of the linear spectrum forced by "analytic reason", they are, in fact, antipodally inter-related and topologically may be glued together in the projective geometric rooting of this linear spectrum, centered around the notion of an obstacle.

In order to articulate this claim, we will consider the dual pair of concepts consisting of ambiguity and information in relation to communication. On the side of the myth, we will scrutinize the narrative referring to the genesis of Goddess Aphrodite according to Hesiod's Theogony. On the side of the mathematical logos, we will scrutinize the theorem referring to the genesis of the roots of a polynomial equation by radicals according to Galois' Theory and its far-reaching articulations. The remarkable conclusion is that in both cases information emerges as "anadyomene" from another level of hypostasis, being in communication with the level where the initial obstruction is localized by means of bidirectional bridges.

Following the narrative of Hesiod's Theogony, the genesis of Goddess Aphrodite took place in an extraordinary manner. She emerged in the form of a fully grown female figure rising out of the sea foam (aphros). Her name Aphrodite, or Aphrogeneia, according to Hesiod, originates from the fact that she grew and formed amid the sea foam. The epithet anadyomene uncovers the metaphora of Aphrodite's genesis. This is a process of circulation between the Heavenly level (Uranus), and the Earthly level (Gaia), which is meant to embrace the obstacle of heterogeneous, chimerical and intrinsically incongruent constituency of these two levels. The initiator of this obstacle-embracing 
communicative process is the Titan Chronos, who stands for the personification of Time as a unitary circular flow reciprocally bridging these two levels into a germinal syzygy.

According to the myth, Chronos forced the transposition of the sperm of Uranus into the sea water, and out of this syzygy a white foam spread around the locus of germination in the sea from the immortal flesh. In this foam there grew the maiden Aphrodite virtualized, to emerge as a fully grown in-formed spectral figure by inverse transposition to the level of Gods, thereby completing the temporally circulating metaphorical process of anadyomene initiated by Chronos. It is important to highlight that the two levels of the myth are not hierarchically ordered, meaning that there is no relation of hypotaxis or subordination of one level to the other.

On the contrary, the two levels exist autonomously in parataxis and only the unitary circular flow of Chronos binds them through reciprocal bridges. The bridge from the Heavenly to the Earthly level is the germination bridge, whereas the reciprocal or conceptually inverse one is the virtual growth bridge. The latter is characterized as virtual because the growth of the figure to emerge as Aphrodite is always surrounded by the sea foam, and thus, the growing figure is indistinguishable and inseparable from the foam.

This is crucial for conceiving, both the symmetry implicated by the circular unitary flow-action of Chronos, and the emergence of Aphrodite as a fully grown in-formed figure only after breaking the symmetry of this inter-level communicative flow. Thus she becomes separated from the sea foam and distinguished or discerned spectrally from it at the level of Gods.

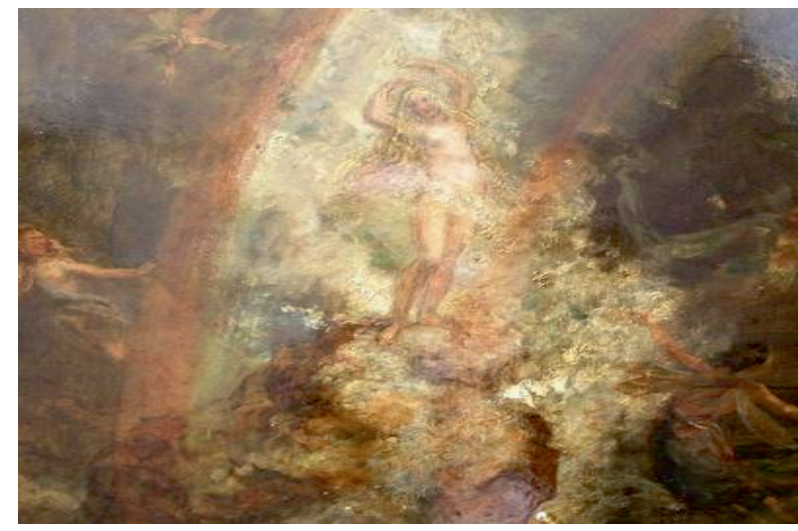




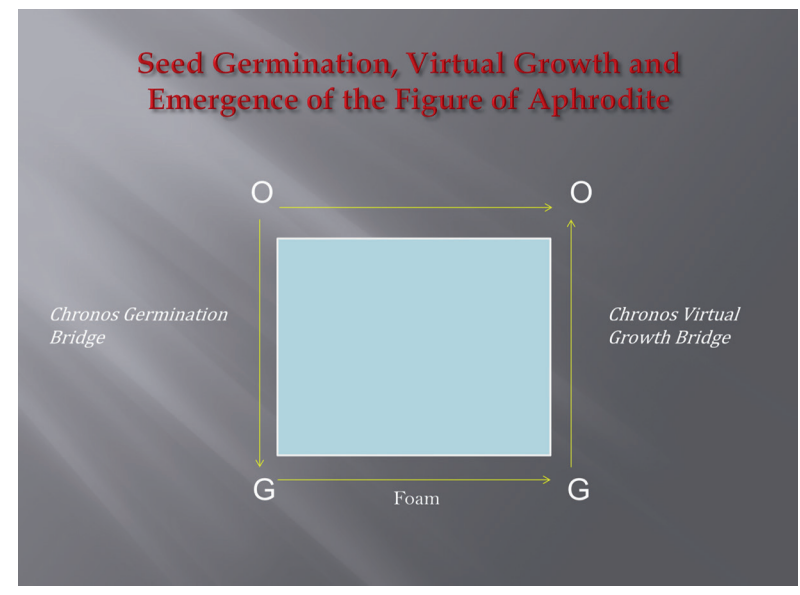

Galois' monumental work focus on the resolution of the problem of algebraic solvability of polynomial equations, i.e. equations of the form $p(x)=0$. Of most interest is Galois' paper entitled "Memoir On the Conditions for the Solvability of Equations by Radicals". In this algebraic treatise, he proves that no general formula for the roots of a fifth, or any higher degree polynomial equation can possibly be found if we employ the usual algebraic operations of addition, subtraction, multiplication, division, and applying radicals, i.e. taking roots of the $n-t h$ degree.

The first novelty of Galois' method consists in the introduction of the group concept, which he devised as a structure $G$ that encodes the permutations of the roots of a polynomial $p(x) \in \mathbb{F}[x]$, which map each root onto a conjugate of it, where $\mathbb{F}$ is a field, called the ground field of coefficients.

The second novelty of Galois' method consists in employing the structure of the above group $G$ together and in relation to the structure of its invariant subgroups under conjugation, in other words, its self-conjugate or normal subgroups. The underlying reason is that the notion of structural divisibility of a Galois group becomes possible only with respect to its normal subgroups.

The third novelty of Galois' method consists in the notion of group solvability. The triumph of Galois theory is based on the theorem that a polynomial equation is solvable by radicals if and only if the corresponding Galois group of the equation is solvable. A solvable group is a group whose derived series terminates in the trivial subgroup. Intuitively, the derived series is a stratification into group levels 
together with a descending staircase among these strata formed by identifying each subgroup in the descending series with a normal subgroup of the previous one. Thus, a group is solvable if by descending into lower and lower subgroup strata by division with normal subgroups we end up with the trivial subgroup.

In this state of affairs, the Galois group $G$ corresponding to a polynomial $p(x) \in \mathbb{F}[x]$, where $\mathbb{F}$ is the ground field, can be though of as a non-numerical, but structural measure of complexity of this polynomial. In turn, the repeated process of division involved in setting up the derived series of $G$, by means of normal subgroups, can be thought of as a process of complexity reduction until the trivial subgroup of $G$ is eventually reached. It is important in this frame of thinking to clarify what we mean by a structural measure of complexity as this is encoded in the Galois group of a polynomial $p(x) \in \mathbb{F}[x]$. The general notion of a group is associated with a criterion of symmetry under its action. What is the implicated notion of symmetry in this case, and how can it be associated with a measure of complexity?

The answer lies in the observation that the Galois group $G$, being the group of permutations of the roots of a polynomial $p(x) \in \mathbb{F}[x]$, which maps each root onto a conjugate of it, where $\mathbb{F}$ is the ground field, actually encodes these $\mathbb{F}$-transformations of the roots of $p(x)$ that cannot be distinguished from the resolution capacity afforded by the ground field $\mathbb{F}$. In other words, the Galois group $G$ of $p(x)$ is an ambiguity group with respect to $\mathbb{F}$, and this ambiguity structure amounts to a measure of complexity of $p(x)$ in relation to the resolving means afforded by $\mathbb{F}$. Henceforth, the symmetry encapsulated by the Galois group $G$ of $p(x)$ in relation to $\mathbb{F}$ can be reduced only by possible successive symmetry reductions, by the process of divisibility with normal subgroups in a descending fashion, until the total symmetry constituting the structural measure of $\mathbb{F}$-complexity is completely reduced.

Reciprocally, the same effect can be achieved by successive extensions of the ground field $\mathbb{F}$ to larger fields that increase the resolution capacity afforded by the ground field itself, until eventually all roots can become distinguishable. More precisely, given a polynomial $p(x) \in \mathbb{F}[x]$ of degree $n$, it can be shown that a minimal extension of $\mathbb{F}$, exists, called the splitting field $\mathbb{F}^{p}$ of $p(x)$, in which all $n$ roots of $p(x)$ can be distinguished (counted with multiplicity). The splitting field $\mathbb{F}^{p}$ of $p(x)$ constitutes in this way the smallest field extension of $\mathbb{F}$ over which the polynomial $p(x)$ splits or decomposes into linear factors. 
For instance, let us consider the classical case of the polynomial $p(x)=x^{2}+1$, i.e. $p(x) \in \mathbb{R}[x]$, where $\mathbb{R}$ is the ground field of coefficients of $p(x)$. We know that this polynomial does not have any roots in the field of real numbers $\mathbb{R}$. In particular, the two roots of the polynomial equation $p(x)=0$, namely $i$ and $-i$, do not belong into the ground field of coefficients of $p(x)$, but can be located in the minimal field extension of $\mathbb{R}$ into $\mathbb{R} \bigoplus i \mathbb{R} \cong \mathbb{C}$. In any case, we also know that the field $\mathbb{C}$ is algebraically closed. In other words, if $\mathbb{D}$ is an algebraic extension of $\mathbb{C}$, then $\mathbb{D}=\mathbb{C}$. Conceptually, the roots $i$ and $-i$ are not distinguishable from the resolution capacity afforded by the ground field $\mathbb{R}$, so that the minimal field extension from the ground field $\mathbb{R}$ to the algebraically closed field $\mathbb{C}$, i.e. $\mathbb{R} \rightarrow \mathbb{C}$, is required to make these two roots distinguishable.

Let us explore now, how the Galois group of the minimal field extension $\mathbb{R} \rightarrow \mathbb{C}$ emerges. There are two equivalent ways to describe it. The first one is the original method devised by Galois, to describe the Galois group of the field extension $\mathbb{R} \rightarrow \mathbb{C}$ as a group of permutations of the roots of the polynomial equation $p(x)=x^{2}+1=0$, which map each root onto a conjugate of it. The second one is the method devised by Richard Dedekind, i.e. to consider the group of automorphisms of the splitting field $\mathbb{C}$, obtained by the minimal field extension $\mathbb{R} \rightarrow \mathbb{C}$, which leaves the ground field $\mathbb{R}$ fixed. We note that an automorphism of this form is a one-to-one and onto homomorphism from $\mathbb{C}$ to itself that leaves $\mathbb{R}$ fixed, or equivalently, invariant. The connection between these two equivalent viewpoints, comes into force if we interpret the Galois group of permutations of roots as a subgroup of a symmetric group. In general, thinking about the Galois group of a polynomial with degree $n$ as a subgroup of the symmetric group $S_{n}$ captures the original viewpoint of Galois, and provides the connection with Dedekind's reformulation of Galois theory.

The symmetric group on $n$ letters is realized as a group of permutations of these $n$ letters, so may identify a symmetric group element $\sigma$ with the corresponding permutation. In general, associating to each element of the Galois group its permutation on the roots of the polynomial, viewed as a permutation of the subscripts of the roots of the polynomial when we list them in a particular order as $\alpha_{1}, \alpha_{2}, \ldots \alpha_{n}$ is an injective homomorphism from the Galois group to the symmetric group $S_{n}$. Two different choices for indexing or ordering the roots of 
the polynomial can lead to different subgroups of the symmetric group $S_{n}$, but they will always be conjugate subgroups.

In general, let $\alpha_{1}, \alpha_{2}, \ldots \alpha_{n}$ be the $n$ distinct roots of $p(x)=0$ in the minimal field extension of $\mathbb{F}$, i.e. in the splitting field $\mathbb{F}^{p}$ of $p(x)$, in which all distinct $n$ roots of $p(x)=0$ can be distinguished. Then the Galois group $G$ permutes these roots. Now, any polynomial expression in these roots, which is left invariant under any permutation of the roots, and thus is a symmetric polynomial expression thereof, is located in the ground field $\mathbb{F}$. The basic examples of such expressions are the elementary symmetric functions in the roots. It is precisely in this sense that the $n$ roots of $p(x)=0$ are indistinguishable from the resolution capacity afforded by the ground field of coefficients $\mathbb{F}$, that is, in terms of all polynomial expressions symmetric with respect to $\mathbb{F}$ in the roots of $p(x)=0$. In other words, and considering the simple case of two roots, the existence of at least one non-symmetric polynomial expression with respect to $\mathbb{F}$, meaning a polynomial expression not preserved by the permutation of these roots, would provide the means to distinguish between them.

We are ready now to come back to our initial problem; how to describe the emergence of the Galois group of the minimal field extension $\mathbb{R} \rightarrow \mathbb{C}$. From Dedekind's perspective, only two automorphisms of the splitting field $\mathbb{C}$ exist, obtained by the minimal field extension $\mathbb{R} \rightarrow \mathbb{C}$, which leaves the ground field $\mathbb{R}$ invariant, namely the identity automorphism $z \mapsto z$, and the complex conjugation automorphism $z \mapsto \bar{z}$. In particular, if $\sigma$ is such an automorphism of $\mathbb{C}$, then it is completely determined by the action of $\sigma$ on $i, \sigma(i)$; i.e. if $\sigma(i)=i$, then $\sigma(z)=z$ for all $z \in \mathbb{C}$, whereas if $\sigma(i)=-i$, then $\sigma(z)=\bar{z}$ for all $z \in \mathbb{C}$. Thus, the Galois group of $\mathbb{C}$ over $\mathbb{R}$ is a group consisting of two elements, namely complex conjugation and the identity map. From Galois' perspective, the Galois group of the field extension $\mathbb{R} \rightarrow \mathbb{C}$ is the group of permutations of the roots of the polynomial equation $p(x)=x^{2}+1=0$, which map each root onto a conjugate of it. There are only two permutations, namely the identity permutation and the complex conjugation permutation, and clearly any polynomial expression in the roots $i$ and $-i$, which is left invariant under any permutation of these two roots, and thus provides a symmetric polynomial expression in these roots, is located in the ground field $\mathbb{R}$. Thus, the ground field $\mathbb{R}$ remains invariant under the action of the Galois group $G$ consisting of the identity and the complex 
conjugation permutation, and therefore, the two roots $i$ and $-i$ are indistinguishable from the resolution capacity afforded by $\mathbb{R}$.

We conclude that the Galois group $G \cong Z_{2}$ of the polynomial equation $p(x)=x^{2}+1=0$ is an ambiguity group with respect to $\mathbb{R}$, and this ambiguity structure, induced by the two possible permutations of the roots in the present case, defines a measure of complexity of $p(x)$ in relation to the resolving means afforded by $\mathbb{R}$. This group-theoretical measure of complexity expresses that the two roots $i$ and $-i$ are indistinguishable from the resolution capacity afforded by $\mathbb{R}$. They eventually become distinguishable only by a process of field extension, which terminates in the minimal field extension $\mathbb{R} \rightarrow \mathbb{C}$, where $\mathbb{C}$ is identified as the splitting field of $p(x)$. In turn, the ambiguity group $Z_{2}$ is identified as the group of automorphisms of the splitting field $\mathbb{C}$, which leaves the ground field of coefficients $\mathbb{R}$ invariant, and thus fixes it.

Let us now consider a general polynomial equation of the form $p(x)=0$. The proper starting-level to settle the question referring to the idea of algebraic solvability of $p(x)=0$ by radicals is the theory of algebraic fields. More precisely, if we start with the ground field of coefficients $\mathbb{F}_{0}:=\mathbb{F}$, we may produce an unfolding sequence of successive field extensions $\mathbb{F}_{0}, \mathbb{F}_{1} \ldots \mathbb{F}_{N}$ by the adjunction of surds, so that eventually $\mathbb{F}_{N}$ emerges as the splitting field for $p(x)$. The theory of algebraic groups, on the other side, due to their multiplication and division properties, set the proper level to examine the structure of such extensions by surds, as follows: When the field $\mathbb{F}_{i+1}$ arises from $\mathbb{F}_{i}$ by the adjunction of the surd $\alpha^{1 / 0}$, then the corresponding group $G_{i+1}$ is a normal subgroup of the group $G_{i}$, with an Abelian factor group. Eventually, one obtains the principal theorem of Galois theory, formulated as follows:

The polynomial equation $p(x)=0$ is solvable by radicals if and only if its Galois group $G$ is solvable, that is if a descending series of groups exists, such that $G_{i+1}$ is a normal subgroup of the group $G_{i}$ with an Abelian factor group, and $G_{0}$ is $G$, while $G_{N}$ consists of the identity alone. 
We stress that the Galois group $G_{0}:=G$ is identified with the group of those automorphisms of the splitting field $\mathbb{F}_{N}$ for $p(x)$ that preserve the ground field of coefficients $\mathbb{F}_{0}:=\mathbb{F}$, and thus they fix it. According to our interpretative scheme, the descending series of groups starting from the Galois group $G_{0}:=G$ until the trivial group $G_{N}$ is eventually reached, represents all the successive stages of symmetry reduction. That is to say all the successive stages of complexity or ambiguity reduction with respect to the ground field $\mathbb{F}_{0}:=\mathbb{F}$ until all the roots of $p(x)=0$ become distinguishable in the inverse ascending series of field extensions from the ground field $\mathbb{F}_{0}:=\mathbb{F}$ to the splitting field $\mathbb{F}_{N}$.

We conclude that an inverse correspondence obtains between the descending series of groups starting from the Galois group $G_{0}:=G$ and terminating at the trivial group $G_{N}$ with the ascending series of fields starting from the ground field $\mathbb{F}_{0}:=\mathbb{F}$ and terminating at the splitting field $\mathbb{F}_{N}$ with respect to any polynomial equation of the form $p(x)=0$ solvable by radicals. The crucial points to notice are the following:

i As the Galois group is the group of all those automorphisms of the splitting field for $p(x)$ that preserve the ground field of coefficients, in the same way a subgroup of the Galois group is a group of automorphisms of the splitting field for $p(x)$ that preserve an intermediate extension of the ground field of coefficients;

ii The internal symmetry or structural complexity reduction of the Galois group takes place by division with a normal subgroup. Intermediate extensions of the ground field corresponding to a normal subgroup of the Galois group are called Galois field extensions;

iii There is a one-to-one correspondence between Galois field extensions and normal subgroups of the Galois group;

iv The Galois group -with normal subgroups structure- corresponds bijectively in an inverse manner to the ground field -with Galois extensions- structure. 


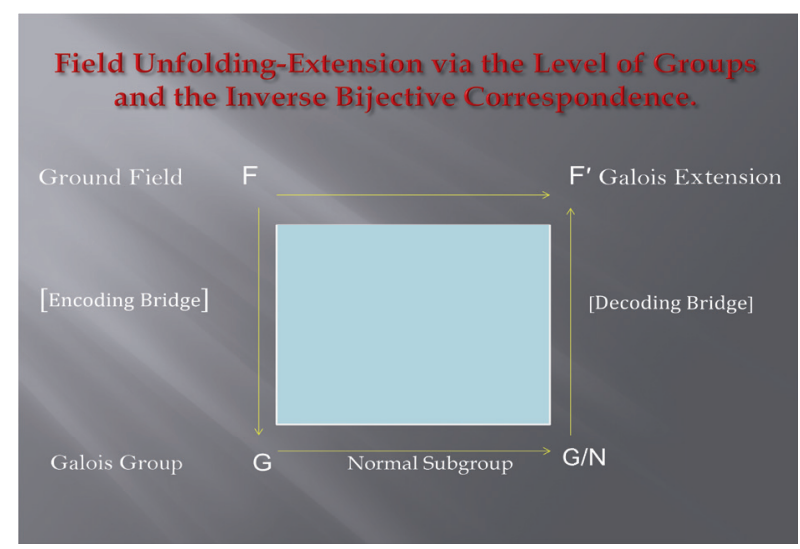

The central conceptual aspect of the so called Erlangen program, conceived by Felix Klein, is expressed by the thesis that the objective content of a geometric theory is captured by the group of transformations of a space. The crucial insight of Klein's program is that transformation groups constitute an algebraic encoding of a criterion of equivalence for geometric objects. Moreover, a transformation group determines the notion of what it is to be a meaningful property of a concrete geometric figure. Therefore, from the Erlangen perspective, a geometric figure may be conceived from an abstract algebraic viewpoint as a manifold acted upon transitively by a group of transformations. The decisive aspect of the criterion of equivalence that a transformation group furnishes is its use in characterizing kinds or types of geometric figures and not particular instances of these figures.

The above leads to the idea that geometry, in an abstract sense, refers to kinds of figures, which are specified by the transformation group of the space. Each kind can have infinitely many instantiations, thus the same geometric form may be manifested in many different ways, or else assume multiple concrete realizations. This reveals an important ontological dimension of Klein's program, since a transformation group of a space provides an efficient criterion to abstract a geometric kind from particular geometric instantiations, whereas the specific details of these instantiations, irrespective of their features as instances of a geometric kind, is irrelevant. In the light of this, a geometry is specified by a group and its transitive action on a space, which remarkably can be presented in a purely algebraic way as a group homomorphism from the transformation group to the group of automorphisms of the underlying space. Conceptually speaking, the form of a geometric theory is encoded in the transitive action of a 
respective transformation group. Different particular geometric configurations are the same in form if and only if they share the same transformation group. In other words, the transitive group action provides a precise characterization regarding matters of geometric equivalence.

Mathematically, the above thesis is expressed as the principle of transference, or principle of isomorphism, induced by a transitive group action on a space. A transfer of structure is taking place by means of an isomorphism providing different equivalent models of the same geometric theory. Philosophically, an Aristotelian conception of space underpins this thought, according to which space is conceived as being matter without form. The form is brought about by the action of a concrete transformation group. Still, more important, the space itself may be considered as the quotient of the transformation group over a closed subgroup of the former. A change in algebraic form, or else, a change of transformation group signifies a change in geometry, in the sense that the equivalence criterion encoded in the group action is altered.

Thus, moving from a group to a larger one amounts to a change in the resolution unit of figures, expressed as a relaxation of the geometric equivalence criterion involved in the procedure. In effect, the criterion of equivalence serves as a powerful classification principle for geometries in relation to group hierarchies. A crucial aspect of the Erlangen program is that it does not specify which underlying manifolds exist as spectra of corresponding observable algebras, but deals with the possible existence of geometric structures on these manifolds in relation to the action of form-inducing transformation groups upon them. This leads naturally to a bidirectional relation of dependent-variation between transformation groups and geometric structures on manifolds. This bidirectional relation conveys the information that two spaces cannot have different transformation groups without differing as geometric structures, whereas the converse is clearly false. GROUP OF LOOPS

In the early 19th century, Jules Henri Poincaré attempted to probe the connectivity problem of a topological space by using paths, and in particular, loops based at a point of this space. This approach gave rise to homotopy theory, and in particular, led to the notion of the fundamental group of a topological space. The fundamental group at a point of a space is defined in terms of the set of based loops at this point modulo homotopies. The notion of homotopy is based on a homeotic criterion of identity of based loops, which is expressed in terms of invariance under continuous distortion and shrinking. 

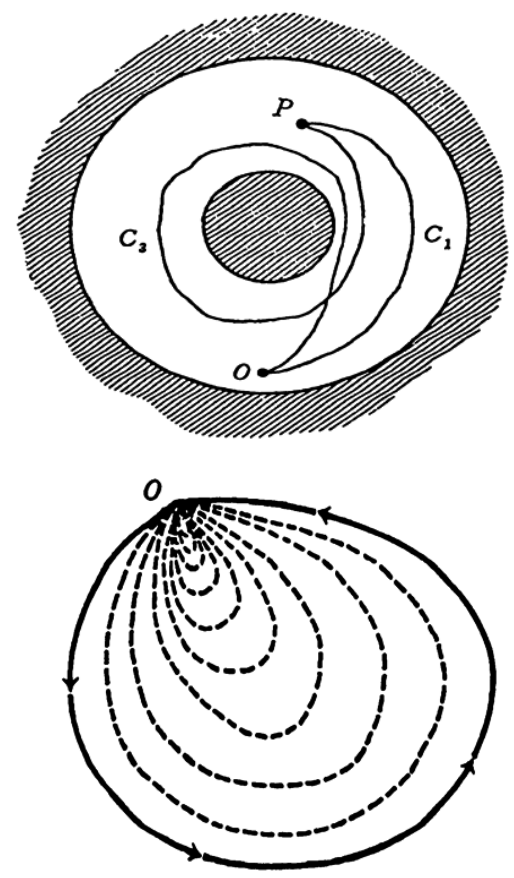

The most basic example is the example of the unit circle $S^{1}$. If we consider homotopy classes of based loops winding around the circle, then they are classified by the number of times winding around the circle. Thus, the fundamental group of the circle is the additive group of the integers $\mathbb{Z}$. If we think of the real line $\mathbb{R}$ as a helix in 3 - $d$ space covering the unit circle $S^{1}$ depicted in 2-d space below, then the continuous surjective group homomorphism $p: \mathbb{R} \rightarrow S^{1}$ is the covering projection, which is given by the exponential map. In other words, the exponential map wraps the real line anticlockwise around the unit circle. We notice that the real line $\mathbb{R}$ is simply connected, thus it is a universal covering space of $S^{1}$. Moreover, the symmetries of $\mathbb{R}$ leaving the circle $S^{1}$ invariant is given by $t \mapsto t+k$, where $k$ in $\mathbb{Z}$ is the winding number, obtaining in this manner the group isomorphism $S^{1} \cong \frac{\mathbb{R}}{\mathbb{Z}}$. 

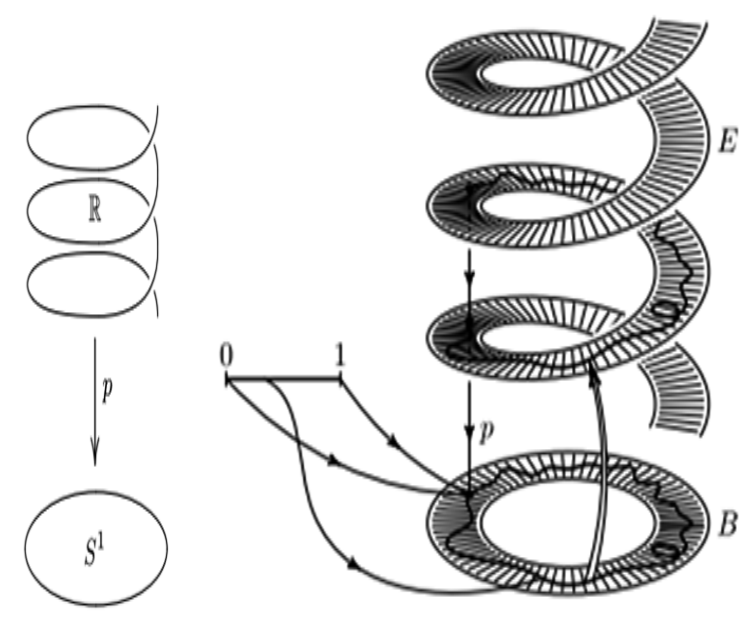

In general, the set of equivalence classes of based loops with respect to continuous distortion and shrinking can be always endowed with a multiplicative group structure under the operation of composition of paths. If the topological space is path-connected, meaning that any two points may be joined by a path, then the isomorphic class of the fundamental group does not depend on the selection of the base point, since the respective fundamental groups at two different base points can be made isomorphic. A path-connected space is always connected. The crucial thing is that it is simply-connected, and thus a geometric space, if it has a trivial fundamental group.

\subsection{DISCRETE FIBRATION: FROM COVERINGS TO THE MONODROMY ACTION}

A covering space of a base topological space is a local homeomorphism, such that for each point on the base space, the inverse image of an open set containing this point is a disjoint union of open sets in the covering space lying over the base, each of which is mapped homeomorphically on this open set, as it is displayed schematically below. In particular, if the base space $X$ is connected, then the fibers of the covering space projection $p: Y \rightarrow X$ are all homeomorphic to the same discrete space $I$, such that locally, $Y$ is isomorphic with $X \times I$. 


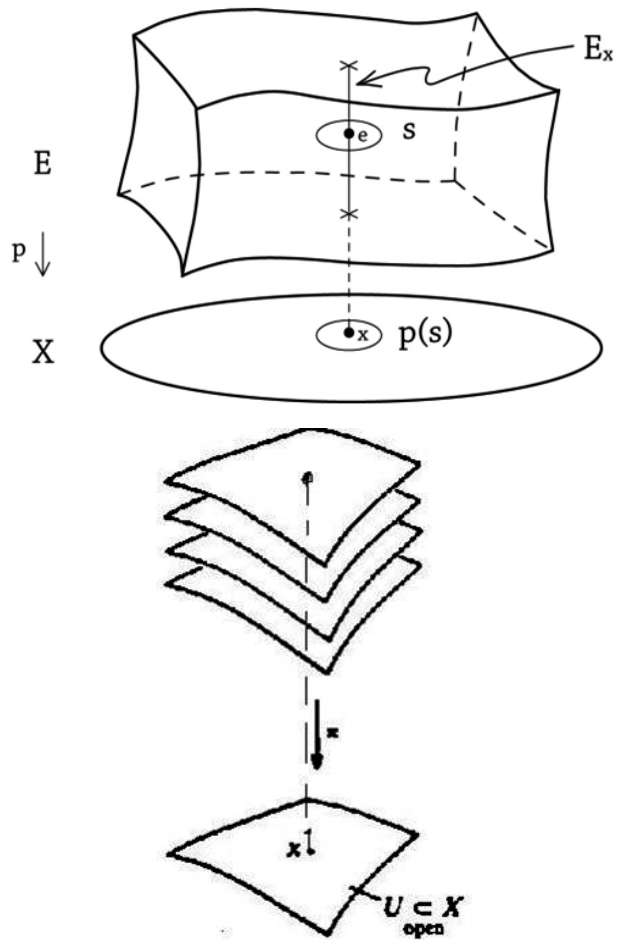

The most important examples of covering spaces arise from group actions on topological spaces. Let $G$ be a group acting continuously from the left on a topological space $Y$. The action of $G$ is even if each point $y \in Y$ has some open neighborhood $U$ such that the open sets $g U$ are pairwise disjoint for all $g \in G$.

We recall that if a group $G$ acts from the left on a topological space $Y$, one may form the quotient space $Y / G$ whose underlying set is the set of orbits under the action of the group $G$ and the topology is the finest one that makes the projection $Y \rightarrow Y / G$ continuous. If $Y$ is connected, then the qualification of the action of $G$ on $Y$ as even, according to the above, makes the surjective projection $Y \rightarrow Y / G$ a covering one, or equivalently, $Y$ becomes a covering space of $Y / G$. For example, let the group $\mathbb{Z}$ act on the space of the reals $\mathbb{R}$ by translations $x \mapsto x+n$. In this case, we obtain $\mathbb{R}$ as a covering space of the space of orbits $\mathbb{R} / \mathbb{Z}$, where the latter is homeomorphic to the circle $S^{1}$.

Given a covering projection $p: Y \rightarrow X$, where the base $X$ is locally connected, we may consider the group of automorphisms of the 
covering space $Y$ compatible with the projection $p$, denoted as $\operatorname{Aut}(Y \mid X)$. We note that for each $x \in X$, the fiber $p^{-1}(x)$ is mapped onto itself under the (left) action of the group $\operatorname{Aut}(Y \mid X)$. The important thing is that if the covering space $Y$ is connected, then the action of the group $\operatorname{Aut}(Y \mid X)$ on $Y$ is an even action. Not only this, but inversely, if $G$ is a group whose action is even on a connected space $Y$, the group of automorphisms of the covering space $Y$ compatible with the projection $Y \rightarrow Y / G$, i.e. $\operatorname{Aut}(Y \mid X)$ is identical with $G$.

The objective posed by Alexander Grothendieck was to explore how Galois's theory can admit a natural instantiation in this context. The initial simple observation is that in the Galois theory of field extensions if we think of the base field as a base point, then a finite separable extension of this field should be thought of as a discrete set of points mapping to this base point. Galois theory then furnishes this discrete set of points with a continuous action of the Galois group which leaves the base point fixed. In this state of affairs, it is a naturally emerging idea to consider as a base not just a point but a more general topological space. The role of field extensions would then be played by certain continuous surjections of the type discussed above, called covering space projections, whose fibers are discrete spaces.

The main thing to highlight in Grothendieck's perspective on Galois theory is that he follows the original Galois conception of the Galois group of a field extension as a group of permutations of the roots of a corresponding polynomial equation, which map each root onto a conjugate of it. Thus, there exists an action of the Galois group of the considered field extension via permutation of the roots of the corresponding polynomial equation. Grothendieck observed that this action is characterized by two important properties. First, it is a transitive action, and second, it is a continuous action if the Galois group is viewed as a topological group. Based on these two properties of the action of the Galois group on the finite set of roots of the polynomial equation, he proved the following correspondence, setting up the ground for generalizing the initial context of application of Galois theory:

There is a one-to-one correspondence between isomorphism classes of finite (separable) extensions of a base field and finite sets equipped with a continuous and transitive action of the Galois group by permutations.

The generalization amounts to introducing the notion of a Galois covering space $p: Y \rightarrow X$ as analogous to the notion of a Galois extension of a base field, which is enforced by considering the even action of the group of topological automorphisms $\operatorname{Aut}(Y \mid X)$ as 
analogous to the action of the corresponding Galois group in the field case. In this manner, a connected covering space $p: Y \rightarrow X$ is qualified as a Galois covering space if and only if the group $\operatorname{Aut}(Y \mid X)$ acts transitively and continuously on each fiber of $p: Y \rightarrow X$ playing the role of a topological Galois group in this setting. Equivalently, we have a Galois covering space if and only if each orbit of the quotient $Y / \operatorname{Aut}(Y \mid X)$ is identical to a whole fiber of $p: Y \rightarrow X$.

Then, the fundamental theorem of Galois theory in the case of (finite) field extensions, according to which, the Galois group-with normal subgroups structure- corresponds bijectively and inversely to the ground field-with Galois extensions structure, can be transcribed in the topological setting as follows:

The topological Galois group -with normal subgroups structurecorresponds bijectively in an inverse manner to the base topological space -with Galois covering spaces structure.

The next step is to examine how the covering principle, fits and gets naturally unified with the Galois metaphora in the context of the topological generalization of Galois theory by Grothendieck, according to the above. Ultimately, the covering principle targets the eventuation of the universal covering space, characterized uniquely by the property of simple-connectivity. This is the key notion in the present case if it can be qualified appropriately through some group theoretic structure that can assume the role of a topological Galois group. Recalling from Poincaré's metaphora that a path-connected topological space is simply-connected if it has a trivial fundamental group, the eventuation of the universal covering space can be transcribed group-theoretically thanks to the notion of the fundamental group of the base space, as targeting the annihilation of the fundamental group of the base.

The annihilation of the fundamental group of the base space can be interpreted as a process of complexity contraction, or symmetry reduction, in the Galois theoretic sense, if and only if there exists an even, transitive and continuous action of the fundamental group on each fiber of the universal covering space $p: Z \rightarrow X$. If this is the case then the fundamental group of the base space actually plays the role of a topological Galois group, whose symmetry is reduced in successive stages by division with normal subgroups corresponding to Galois covering spaces; Ultimately the whole symmetry is completely reduced by unfolding to the universal covering space characterized by simple-connectivity, which thus bears a trivial fundamental group.

In other words, the maximal ambiguity engulfed in the fundamental group of the base space at a marked point, interpreted as a structural measure of multiple-connectivity characterizing the homotopic complexity of the base at this point due to its prospective qualification as a topological Galois group, can be entirely eliminated by 
unfolding to the simply-connected universal covering space of the base topological space.

It remains to examine if there actually exists an even, transitive and continuous action of the fundamental group on each fiber of the universal covering space $p: Z \rightarrow X$, qualifying it as a topological Galois group. The fact that such an action actually exists is due to the two most important properties of a covering space, namely the path-lifting property and the homotopy-lifting property from the base to the fibers of a covering space.

The main idea is that both paths and loops (belonging to a homotopy class of the fundamental group) on the base space can be lifted uniquely from the base to the fibers of a covering space. In particular, if we consider a based loop at a marked point of the base space, then its unique lift on a covering space is not necessarily a loop, but it is always going to be a path whose starting and ending point belong to the same fiber of the covering space that projects to the marked point of the base, where the loop is based. Since such a based loop is an element of a homotopy class in the fundamental group at the marked point, the transition from the starting to the ending point of the fiber over the marked point induced by the lift of this based loop, amounts to an even, transitive, and continuous action of the fundamental group at the marked point on the fiber of the covering space over this point. This action is called the monodromy action of the fundamental group (at the marked point) on the fiber of the covering space over this point.

Grothendieck proved that the monodromy action actually gives rise to a functor from the category of covering spaces over the base space $X$, where a marked point has been depicted, towards the category of sets equipped with a left action of the fundamental group at this point. This takes place by sending a covering space $p: Y \rightarrow X$ to the fiber $p^{-1}(x)$ over this marked point, called the fiber functor at the marked point. Essentially the fiber over a marked point encapsulates the global connectivity depth of this point in the presence of topological obstacles.

There is a subtle indirect self-referential metaphora regarding the notion of a point in a topological space that is worth explicating. More precisely, the notion of a point in a topological space is only implicitly assumed, and thus it needs to be articulated through the basic topological characteristic, which is connectivity in the presence of obstacles. The metaphora from the topological domain of connectivity to the algebraic domain of the fundamental group, culminating in the notion of a covering space, pertains to the level of points by means of the monodromy action that eventually indirectly articulates a point through its fiber.

The fiber functor at a marked point of the base space, induces the following categorical equivalence: 
There exists an equivalence between the category of covering spaces over the base space $X$ and the category of sets equipped with a left action of the fundamental group at the marked point, such that connected covering spaces correspond to sets with a transitive left action of the fundamental group at the marked point, and Galois covering spaces correspond to coset spaces of normal subgroups of the fundamental group at the marked point.

In this way, we obtain the following unification of the covering principle with Galois theory in the current topological setting:

The fundamental group of a base topological space (where a marked point has been depicted) -with normal subgroups structurecorresponds bijectively in an inverse manner to the base topological space -with Galois connected covering spaces structure-.

The only condition for the validity of Grothendieck's theorem, establishing the role of the fundamental group as a Galois group in the setting of connected covering spaces, is that the base space is connected and each point of this space has a basis of simply connected open neighbourhoods.

Now, if the Galois covering space is a universal simply-connected covering space of the base space $X$ where a marked point $x$ has been depicted, denoted by means of the covering projection $p: \widehat{X}_{x} \rightarrow X$, then it corresponds to a set equipped with a left transitive action of the whole fundamental group at this point $\pi_{1}(X, x)$, or else a $\pi_{1}(X, x)$-set. This action is of the Galois-type since it induces permutations of all the elements of the universal covering space fiber projecting to the marked point $x$ in the base. Thus, conceptually, it is naturally isomorphic with the group of automorphisms of the universal covering space $\widehat{X}_{x}$ compatible with the projection $p$, denoted as $\operatorname{Aut}\left(\widehat{X}_{x} \mid X\right)$. In this way, the Galois action of $\operatorname{Aut}\left(\widehat{X}_{x} \mid X\right)$ of the universal covering space fiber projecting to the marked point $x$ in the base is identified with the monodromy action of the fundamental group (at the marked point) on the fiber of the universal covering space over this point. 


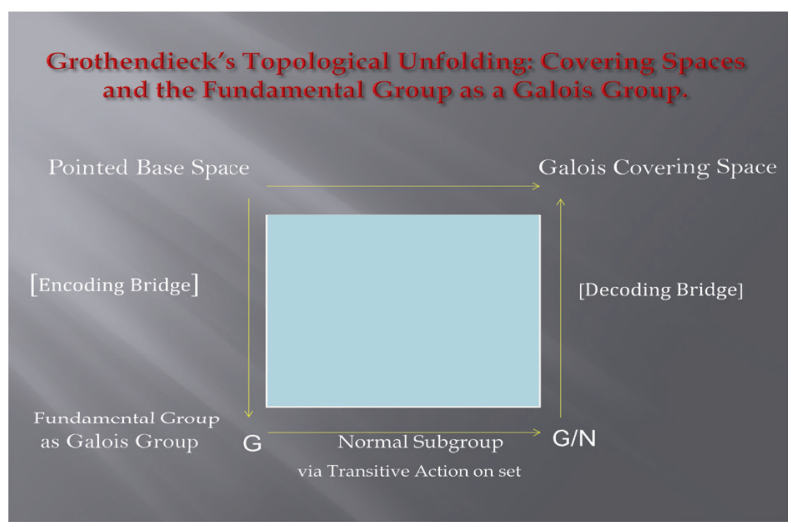
TO THE GLOBAL

The concept of a sheaf is based on two fundamental pillars. The first refers to the notion of a locally defined element of an algebraic structure, whence the second refers to the gluing or pasting conditions of these locally defined elements together. The concept of locality is introduced by means of an appropriate topology, or more generally, in terms of an appropriate covering system. The sheaf is understood as ubiquitious to address the precise manner in which locally defined elements, organized in terms of the structure of groups, or modules, or algebras, or even sets, can be topologically extended from the local to the global. They are thus collated compatibly into global elements over a partially ordered covering system, like the one defined by the open covers of a topological space.

In this sense, a sheaf may be thought of as a continuously variable algebraic structure, whose continuous variation is expressed in terms of its sections over the local covers of a global topological space. The existence of the latter is only implicitly assumed, since the actual objective of a sheaf is the topological articulation of the points of this space through the compatible amalgamation and extendibility of the sections from the local to the global. Thus, essentially the notion of a sheaf targets the nature of points in a topological space in the presence of obstacles to connectivity.

The amalgamation, this process of gluing, is conducted by the formation of appropriate equivalence classes, called germs of sections of a sheaf, which are defined over a partially ordered family of covers of the implicitly assumed global topological space. A section of a sheaf, which is defined over a local cover may be thought of as a partially defined, continuously variable functional relation, whose degree of specificity depends on the spectral resolution afforded by the corresponding cover. 
One of the most interesting aspects of the sheaf concept in comparison to the former set-theoretically articulated notion of a topological space is that a sheaf actually captures the model of a topological space in its spectral becoming. Given that a topological space, for instance a manifold, is physically utilized to represent the event or state structure of a physical theory corresponding to the evaluation of observables, its associated sheaf model pertains to its actual taking place, i.e. to its actual constitution by amalgamating compatibly local observables into global ones. This is because the underlying notion of a global topological space, referring to its structure of points, both standard and singular, is initially only implicitly assumed, and then, step-by-step induced indirectly by completion via the germs of the sheaf. This culminates in extracting the invariant information pertaining to these germs after their integration.

In this sense, we should emphasize that a sheaf model of a space does not have a local structure defined by its points, which are assumed to be absolute and pre-existing according to former set-theoretic lines of reasoning. In contrast, the local structure is considered as being intrinsically continuous with respect to the partially ordered covers of this space. A closed partially ordered family of intersecting local covers, capable of gradually unraveling the invariant information that germs bear at a point, acquires the meaning of a temporal order. The algebraic structure of germs at a point subsumes in this way all the contextual information in relation to this point.

The sheaf completion property at a point is tantamount to the integration of these germs, though of as expressing differentially the local or infinitesimal variations around this point. Eventually, this is precisely the spectral resolution process that characterizes not only the underlying point itself sheaf-theoretically, but also its genetic constitution and variation in relation to the considered temporal order. This is because the global characterization of a point, for example of a physical state or an event, is the final result, the ichnography, or technically the trace of a continuous unfolding process of genetic constitution in terms of granular elements. These granules are precisely the germs of sections around that point, each one of which bears the resolution capacity of their cover-horizon. Therefore, germs incorporate all their compatible subcovers within the pertinent temporal order.

Since Jean Leray's initial conception of the notion of a sheaf, and Grothendieck's articulation of the same concept, the various examples and applications of sheaves have come to play a major role in such diverse fields of mathematics as several complex variables, algebraic geometry, algebraic topology, and differential geometry. This is especially true for Grothendieck's contribution, which generalized the notion of sheaves beyond the realm of topological spaces, making it applicable over general category-theoretic sites. In sum, categories 
equipped with covering systems, called Grothendieck topologies, has created a wealth of new models enriched with the power of the methods of homological algebra.

The algebraic topological ubiquity of the sheaf concept is based on the realization that, since it is possible to localize standard algebraic notions, such as homomorphism, kernel, image, subobject and quotient object sheaf-theoretically, in such a way that these concepts have essentially the same meaning as in abstract algebra, one can interpret them from a categorical standpoint, and infiltrate all the constructions of homological algebra through sheaf theory. The resulting category of sheaves has the same classical properties as the category of Abelian groups, or the category of modules. More precisely, one can define for sheaves direct sums, direct products, tensor products, inductive limits, and all other related concepts.

For this reason, the apparatus of sheaf theory is able to penetrate into various fields of mathematics providing an effective algebraic tool especially in those areas which ask for global solutions to problems whose hypotheses are local. This is due to the fact that there is a natural definition of the cohomology of a site, that is, in the simplest case of a topological space with coefficients in a sheaf. In particular, Grothendieck's insights and formulations led to the crystallization of the idea that the natural argument of a cohomology theory is a pair consisting of a global topological space, or more generally a category-theoretic site, together with a sheaf of coefficients defined over it, rather than just the space itself. In point of fact, and since the topological space is only implicitly assumed, the major role is played by the means of measuring and spectrally resolving this space over its local covers, i.e. by the pertinent sheaf of coefficients. This realization has been transferred to the field of complex analysis, and more recently to the field of differential geometry by the development of the geometric theory of vector sheaves, which are equipped with a connection, according to the framework of Anastasios Mallios, called Abstract Differential Geometry (ADG). Since then, it is a common topos that the sheaf gauge of algebraic-topological localization and extendibility sought-after is always provided by sheaf cohomology.

Cohomology has been invented as an efficient algebraic technique of assigning global invariants to a topological space, or more generally, to a categorical site, for the purpose of capturing group-theoretically its most important shape-related characteristics in a homotopy-invariant way. In particular, the cohomology groups encode the global obstructions for extending sheaf sections from the local to the global level, for example, extending local solutions of a differential equation to a global solution.

For instance, Georges de Rham cohomology theory measures the extent that closed differential forms fail to be exact, and thus, it qualifies 
the obstruction to integrability group-theoretically, since according to the lemma of Poincaré, every closed differential form is locally exact. The de Rham theorem asserts that the homomorphism from the de Rham cohomology ring to the differentiable singular cohomology ring, which is given by the integration of closed forms over differentiable singular cycles, is a ring isomorphism. The sheaf-theoretic understanding of this deep result came after the realization that both the de Rham cohomology and the differentiable singular cohomology are actually special isomorphic cases of sheaf cohomology with values in the constant sheaf of the reals. In particular, it has been also clarified that the de Rham cohomology of a differential manifold depends only on the property of paracompactness of the underlying topological space.

Aside from the cohomological machinery associated with the algebraic theory of sheaves, the general process of transition from the constant to the variable takes place by substituting global rigid set-theoretic algebraic structures with localized continuous sheaf-theoretic algebraic structures. It is in this precise sense that a sheaf may be thought of as a continuously variable set, whose continuous variation is enacted over the employed local covers of a topological site, such that local sections bear the capacity to interlock together in their extendibility from the local to the global. These local covers are required to obey certain topological closure conditions, which in fact, generalize the definition of a topology formulated in terms of open sets covering a classical topological space.

From a physical standpoint, we are forced to consider the conditions of localization of physical observability, to elevate observables from the topos of sets to an appropriate topos of sheaves with respect to a covering system of a global site. The idea is that the global is not directly accessible, and thus, information can only be qualified and quantified sheaf theoretically, i.e. in terms of pasting conditions, from local measurements taking place over the covers of this site. Intuitively speaking, this amounts to a new type of relativization pertaining to the local behavior of observables as opposed to their classical point behavior due to the obstacle erected by objective indistinguishability, or intrinsic randomness, or non-subjective uncertainty.

It is useful here to recall that in the guiding case of a topological space, the notion of a topology provides the means to talk about what a continuous function is between topological spaces. A function is said to be continuous if and only if the inverse image of every open cover of the range is an open cover of the domain topological space. This formulation is an attempt to capture the intuition that there are no breaks or separations in a continuous function. This being so, it is instructive to highlight the following: 
The definition of a topology on a space is solely used for the formalization of what a continuous function is on that space, the continuity of a function is a property which is determined locally, that is only by reference to the open covers of a space.

This means that due to the variability of open covers in the topology the property of continuity of a function should respect the inverse algebraic operations of restriction and unique extension with respect to the open covers of a covering system. Thus, a continuous function can be restricted consistently to open subcovers of any open cover in the topology and inversely extended by gluing uniquely together all its local restrictions. This is the crucial conceptual insight in relation to the notion of continuity that is incorporated in the technical definition of a sheaf.

The above insight referring to the precise formulation of the property of continuity may be generalized in two directions: Firstly, instead of open covers of a topological space we may consider generalized covers under the constraint that they collectively obey topological closure conditions analogous to the ones used for open covers. Secondly, instead of functions varying continuously over local covers, we may consider generalized functional relations, which are precisely the sections of a sheaf. We note that local sections of a sheaf depict functional relations relatively to a local cover. From this viewpoint, a sheaf is essentially the totality of its sections, comprehending both the local and the global in case that the latter actually exist.

Thus, in terms of sections, what actually matters is their consistent interrelation as well as their respective pairwise interlocking properties with respect to the local-global distinctions subsumed by the underlying covers. In particular, the operation of restriction of sections is meaningful with respect to nesting local covers under intersection, whereas the operation of extension or gluing of sections is meaningful with respect to compatible pairwise intersections of sections over their respective local covers, followed by extendibility of a section by another section over the union of their covers. We note that if only the compatibility property of sections under restriction for nested local covers is satisfied, then we obtain a weaker structure called a presheaf. In cases where the compatibility property of sections under extension is also satisfied, then we obtain a separated presheaf. Only in case that the operation of extension results in a unique gluing of sections, that is local sections can be uniquely glued together, a separated presheaf becomes a sheaf.

As a result, in general, there will be more locally defined or partial sections than globally defined ones, since not all partial sections need be extendible to global ones. Nevertheless, a compatible family of partial sections uniquely extends to a global one, or in other words, any 
presheaf uniquely defines a sheaf. More precisely, for the leading example of localization with respect to the open covers of a topological space we have the following basic notions:

A presheaf $\mathbb{F}$ of sets on a topological space $X$, is constituted as an information structure in relation to this space, as follows:

i For every open set $U$ of $X$, there is defined a set of elements denoted by $\mathbb{F}(U)$; and

ii For every inclusion $V \infty U$ of open sets of $X$, there is defined a restriction morphism of sets in the opposite direction:

$$
r(U \mid V): \mathbb{F}(U) \rightarrow \mathbb{F}(V)
$$

such that:

$$
\begin{array}{ll}
\text { a } & r(U \mid U)=\text { identity at } \mathbb{F}(U) \text { for all open sets } U \text { of } X \text {; and } \\
\text { b } & r(V \mid W) \circ r(U \mid V)=r(U \mid W) \text { for all open sets } W \infty V \infty U .
\end{array}
$$

Usually, the following simplifying notation is used: $r(U \mid V)(s):=\left.s\right|_{V}$.

A presheaf $\mathbb{F}$ of sets on a topological space $X$, is defined to be a sheaf if it satisfies the following two conditions, for every family $V_{a}$, $a \in I$, of local open covers of $V$, where $V$ open set in $X$, such that $V=\cup_{a} V_{a}$ :

i Local identity axiom of a sheaf: Given $s, t \in \mathbb{F}(V)$ with $\left.s\right|_{V_{a}}=\left.t\right|_{V_{a}}$ for all $a \in I$, then $s=t$; and

ii Gluing axiom of a sheaf: Given $s_{a} \in \mathbb{F}\left(V_{a}\right), s_{b} \in \mathbb{F}\left(V_{b}\right), a, b \in I$, such that:

$$
\left.s_{a}\right|_{V_{a} \cap V_{b}}=\left.s_{b}\right|_{V_{a} \cap V_{b}}
$$

for all $a, b \in I$, then there exists a unique $s \in \mathbb{F}(V)$, such that: $\left.s\right|_{V_{a}}=s_{a} \in F\left(V_{a}\right)$ and $\left.s\right|_{V_{b}}=s_{b} \in F\left(V_{b}\right)$.

If we consider the partial order of open covers of a topological space $X$ as a category denoted by $\mathcal{O}(X)$, where all arrows are inclusions, then $\mathbb{F}$ denotes the contravariant presheaf/sheaf functor that assigns to each open set $U \subset X$ a set in the category Sets. We 
note that the above definitions hold if instead of presheaves/sheaves of sets we consider presheaves/sheaves of algebraic structures, for example groups, algebras over a field, vector spaces over a field or modules over an algebra.

As the most basic example, if $\mathbb{F}$ denotes the contravariant presheaf functor that assigns to each open set $U \subset X$, the commutative and unital algebra of all real-valued continuous functions on $U$, then $\mathbb{F}$ is actually a sheaf. This is clear since the specification of a topology on $X$, and hence, of a topological localization system on $\mathcal{O}(X))$, is solely used for the definition of the continuous functions on $X$, to be thought of physically as observables on $X$.

Thus, the continuity of each function can be determined locally. This means that continuity respects the operation of restriction to open sets, and moreover that continuous functions can be collated in a unique manner, as is required for the satisfaction of the sheaf condition. More precisely, the sheaf condition in this case means that the following sequence of commutative algebras is left exact;

$$
0 \rightarrow \mathbb{F}(U) \rightarrow \prod_{a} \mathbb{F}\left(U_{a}\right) \rightarrow \prod_{a, b} \mathbb{F}\left(U_{a} \bigcap U_{b}\right)
$$

Let us further assume implicitly that $x$ is a point of a topological space $X$. Moreover, let $T$ be a set consisting of open covers of $X$, containing $x$, such that the following condition holds: For any two open covers $U, V$, containing $x$, there exists an open cover $W \in T$, contained in the intersection $U \bigcap$. We may say that $T$ constitutes a basis for the system of open covers around $x$. We form the disjoint union of all $\mathbb{F}(U)$, denoted by;

$$
\mathbb{D}(x):=\bigcup_{U \in T} \mathbf{F}(U) .
$$

Then we can define an equivalence relation in $\mathbb{D}(x)$, by requiring that $f \sim g$ for $f \in \mathbb{F}(U), g \in \mathbb{F}(V)$, provided that they have the same restriction to a smaller open cover contained in $T$. Then we define;

$$
\operatorname{colim}_{T}[\mathbb{F}(U)]:=\mathbb{D}(x) / \sim_{T}
$$

It is clear that the above definition is independent of the chosen basis of open covers $T$, and thus corresponds to an inductive limit construction. The inductive limit obtained is denoted by $\mathbb{F}_{x}$, and referred to as the 
stalk of $\mathbb{F}$ at the point $x \in X$. We identify an element of $\mathbb{F}$ of sort $U$ with a local section of $\mathbb{F}$ over the open cover $U$.

Then the equivalence relation, used in the definition of the stalk $\mathbb{F}_{x}$ at the point $x \in X$ is interpreted as follows: Two local sections $f \in \mathbb{F}(U), \quad g \in \mathbb{F}(V)$, induce the same contextual information at $x$ in $X$, provided that they have the same restriction to a smaller open cover contained in the basis $T$. Then, the stalk $\mathbb{F}_{x}$ is the structure containing all contextual information at $x$, that is the structure of all equivalence classes.

Moreover, the image of a local section $f \in \mathbf{F}(U)$ at the stalk $\mathbb{F}_{x}$, denoted by $f_{x}$, that is the equivalence class of this local section $f$, is precisely the germ of $f$ at the point $x$. We deduce that the fibration corresponding to a sheaf of sets $\mathbb{F}$ is a topological bundle, called an étale bundle, defined by the continuous mapping $\varphi: F \rightarrow X$, where;

$$
\begin{gathered}
F=\bigcup_{x \in X} \mathbb{F}_{x} \\
\varphi^{-1}(x)=\mathbb{F}_{x} .
\end{gathered}
$$

The mapping $\varphi$ is locally a homeomorphism of topological spaces. The topology in $F$ is defined as follows: for each local section $f \in \mathbb{F}(U)$, the cover $\left\{f_{x}, x \in U\right\}$ is open, and moreover, an arbitrary open cover is a union of covers of this form. Obviously, the same arguments hold in the case of a sheaf of sets $\mathbb{F}$ endowed with some algebraic structure, for example $\mathcal{F}$-algebras (where $\mathcal{F}$ is a field). Finally, the sheaf $\mathbb{F}$ can be canonically identified as the sheaf of cross-sections of the corresponding étale bundle $F$.

We stress that the notion of a sheaf depends on the fact that we require the gluing condition with respect to all covers of any local cover. In principle, one could select some covers of a local cover and require the gluing condition only with respect to the selected covers. In this way, the notion of sheaf would be meant with respect to the selected family of covers. On the other hand, there is no restriction in considering only hereditary or genetically descending covers, that is covers containing all their subcovers. More precisely, any cover can be made hereditary (by adding to each cover all its considered subcovers), and compatible (uniquely glued) families of sections on the original cover are in bijective correspondence with compatible families of sections on the new one. 
This fact provides an important insight on the nature of the topological localization process of observables implicated by the sheaf concept. First, let us think of a local cover as a partial information carrier. Then, the idea of considering hereditary covers can be implemented according to a procedure of refining the resolution grain of information via localization through sieving. More concretely, a sieve on a local cover can be thought of as consisting of spectral horizons distributed across different nested layers, such that, every partial information which is compatible with respect to one of these horizons passes through it but not otherwise.

The conception of covering sieves as hereditary covers used for localizing, and thus sharpening, information with respect to intersecting local covers at deeper ordered depths, gives rise to localization systems of global observable algebras which induce a semantic transition of events from the set-theoretic to the sheaf-theoretic level. In this manner, events are just the traces of the process of sharpening observable resolution by the way of sheaf-theoretic localization. The operation which assigns to each local cover a collection of covering sieves satisfying the appropriate transitivity and stability properties under localization into deeper ordered depths, defines a topology, which is technically called a Grothendieck topology.

The notion of a Grothendieck topology formulated in terms of covering sieves is significant for the following reasons: First, it elucidates precisely the topological concept of locality in relational order-theoretic terms, such that this concept becomes distinguished from its usual spacetime connotation. Second, it permits the amalgamation of local information into global by utilization of the notion of sheaf with respect to the defined topology.

In more detail, the extension of observable information from the local to the global takes place through a compatibly glued family of sections over a covering sieve constituted of local covers of sharper and sharper resolution, giving rise to an inductive localization system upon its closure that ia tantamount to the completion of the localization process. A sheaf assigns a set of sections to each local cover of a localization system. A selection of sections from these sets, one for each local cover, forms a compatibly glued family with respect to a localization system, if the selection respects the operation of restriction, and additionally, if the sections selected agree whenever two local cover of the localization system overlap. If such a locally compatible selection of sections extends uniquely to a global one, then the sheaf conditions are satisfied. We note that in general, there will be more local sections than global ones (if they exist), since not all local information need be extendible to global ones, but a compatible family of local information uniquely extends to a global one with respect to a localization system. It is crucial that sheaf-theoretic localization takes place in terms of 
continuous granular elements, i.e. the sections of a sheaf, which gradually, and in terms of the temporal order they are associated with, expressed by a closed sieve of covers, genetically unfold the becoming of point-events.

It is essential to clarify that the sheaf model of a space takes place in three steps, after the specification of a covering sieve: The first step organizes compatibly the local cover-infiltrated information of sections, such that the latter can be compatibly restricted from the global to the local. This process produces a structure that is only a presheaf. Note that conceptually the implicitly assumed global, from the presheaf-theoretic viewpoint, refers to the global in its potentiality to germinate in the context of some temporal order, and eventually produce facts. The second step involves the process of functional localization of the sections of the presheaf. This is necessary because the sections of the presheaf are not a priori functional elements. It is accomplished by means of the topological realization of the presheaf of sections as a display space constituted by disjoint stalks of germs over each point of the underlying base space. The third step involves the completion of the presheaf, or equivalently the completion of its associated display space, such that the germs belonging to each stalk can be evaluated and produce a fact, temporalizing in this way the pertinent point of the stalk, by disclosing genetically the temporal order they descent from. In this way, it is realized a sheaf, incorporating all the necessary and sufficient conditions for the bidirectional compatibility of information under restriction or reduction from the global to the local, and inversely under unique extension or induction from the local to the global. The latter sheaf-theoretic conceptualization of the global refers now to this term in its actual propensity to explicate points globally in terms of events following the germination of sections with respect to the temporal order they participate and descend from.

Clearly, both of these connotations depend on the role of the covers of a covering sieve, since it is true both, that the production of facts is pre-conditioned by their existence, and their interconnection is possible only via the sheaf-theoretic gluing procedure conducted over these covers. Hence, the local covers can be thought of as both, localities of contextualized potentiality (under restriction from the global to the local), and as localities of relativized facticity (under extension from the local to the global), with respect to which point-events are localized, actualized, and eventually, interconnected consistently.

Due to the dual articulating role of the term global, in relation to both potentiality and actuality, pertaining to the conception of the sheaf model of a topological space as a complete presheaf, according to Leray, a logical scheme of indirect self-referentiality is induced, whence locally all the information is sieved through an appropriate family of local covers. The uniquely defined sheaf-theoretic extension from the local to 
the global takes place thanks to the formation of germs, i.e. equivalence classes of locally or partially defined section compatible by our criteria. Due to the process of extensive connection and the formation of continuous histories in terms of germs in purely topological terms (without assuming any pre-existing metrical linear sequence of an external time line), the notion of a sheaf resolves the self-referentiality induced by the dual role of the topological global in a spiral-like form. The two possible ways of orientation on this spiral-like form represent the inverse processes of ascending (extending from the local to the global) and descending (restricting from the global to the local). Ascent corresponds to indirect self-referential constitution of the global in terms of events, from the local, via the extensive connection of their corresponding germs. This means that the global (in actuality) can be genetically and connectively, that is, historically, accessed only through the germs of observables over the corresponding point-events. Descent corresponds to indirect self-referential resolution of the global (in potential) with respect to a multiplicity of local covers, such that the inversely constituted global (in actuality) achieved by evaluating germs, is compatible with the former. The basic idea pertaining to the above may be simply put as follows: Since the evaluation of a section over a cover gives rise to an observed event, this event is simultaneously implicitly correlated with a germ, i.e. with the whole family of sections, which are compatible with the considered one under restriction, or more generally, under pulling back. Thus, we can access the historicity of an event from a topological perspective through the connective extension of a continuous germ, which indirectly correlates this event to all antecedent events.

Geometric function theory on a Riemann surface, culminating in the thriving field of complex analysis, traces its germ of conception and initial development back to Bernhard Riemann's principles for dealing with the notion of a multiply-extended or multi-fold magnitude. This notion, called simply today a multi-valued function, takes hold because the analytic continuation of a given holomorphic function element along different paths on the complex plane, due to the presence of an obstacle, leads to different branches of that function.

The basic idea of Riemann, giving rise to the covering principle, consists in the replacement of the initial domain of definition of the function with a multiple-sheeted covering of the complex plane, or more generally, of the Riemann sphere, so that this function becomes eventually single-valued on the universal covering space of the initial domain. In this manner, a multiply-extended variable magnitude on the complex plane is unfolded into a simply or uniformly-extended variable 
magnitude on the universal covering space. Thus, the covering principle is essentially based on the idea of metaphora from an obstacle-laden domain to the universal covering of this domain. The latter is qualified as the maximal, simply-connected and obstacle-free unfolding space, where uniform extendibility of an equivalence class, a locally-defined germ of a holomorphic function element, becomes feasible.

Uniform extendibility in the universal covering or unfolding space is thought of in terms of the process of analytic continuation of a multi-valued function along loops surrounding obstacles. Riemann conceived of an infinitely thin sheet propagating along a loop of this kind such that, when one returns to the starting point, one arrives on a different sheet whenever the value of the function obtained by analytic continuation is different from its initial value.

In this way, by performing the analytic continuation along all the possible loops, one may associate a many sheeted smooth compact surface which covers the Riemann sphere with the given multi-valued function, where the former functions become uniform and single-valued. If the covering surface is constructed so that it has as many points lying over any given point in the complex plane (or the Riemann sphere) as there are function elements at that point, then on the universal covering Riemann surface, the analytic function unfolds completely and becomes single-valued. This process of unfolding a multi-valued function by means of spreading out into a multiplicity of branches constituting the universal covering is called the ramification of a multi-valued function.

Consequently, according to Riemann's covering principle, when a multi-valued function unfolds, the covering surface will also unfold with it. In a region where two or more unfoldings of the function occur, the covering surface will be double or multiple. It will consist of two or several sheets, each one of them corresponding to a branch of this function. Around a ramification point of the function, a sheet of the covering surface will unfold to another sheet, and in such a way that, in the neighborhood of this point, the surface may be thought of as a helicoid whose axis is perpendicular to the complex plane at that point. But when, after several windings around the ramification value, the function takes back its initial value, one must assume that the superior sheet of the surface connects to the inferior one by traversing the rest of the sheets. At each point of a surface which represents the way it ramifies, the multi-valued function admits a single determined value, and may therefore be looked upon as a perfectly determined function of the place (of a point) on this surface. It is important to highlight that the different covering branches can be joined together only at points, not along lines.

In modern terminology, a covering Riemann surface gives rise to a holomorphic map with codomain the complex plane, or the Riemann sphere, which is characterized topologically as a ramified covering 
projection or, equivalently, as a branched covering space of the latter. In other words, it is a local homeomorphism, such that for each point on the plane or the sphere, the inverse image of an open set containing this point is a disjoint union of open sets in the covering Riemann surface, each of which is mapped homeomorphically on this open set. This holds universally with the exception of the ramification points, which are inverse images of branch points on the plane or the sphere. At these points, the various branches of the covering Riemann surface, thought of as a helicoid winding around the various corresponding branch points, are alternated or interchanged in winding cycles.

We note that if we forget the fact that these concrete surfaces are spread out over the complex plane (or the Riemann sphere) as branched covering spaces referring to the process of unfolding of a many-valued function, we obtain the notion of an abstract Riemann surface, defined as a two-dimensional real analytic manifold equipped with a holomorphic structural atlas, which is considered as the natural domain of definition of analytic functions in one complex variable. In this manner, the notion of a concrete covering Riemann surface is used only with reference to a many-valued function. Recalling Weyl's suggestive formulation, a concrete Riemann surface is not merely a visual representation of a many-valued function. Quite the opposite, it must throughout be thought of as the prius, as the mother earth in which functions, like plants, can first of all grow and flourish.

If we recall the complex exponential covering projection from $\mathbb{C}$ to $\mathbb{C}^{*}=\mathbb{C}-\{0\}$ :

$$
\exp (z): \mathbb{C} \rightarrow \mathbb{C}^{*}
$$

then, a determination of its inverse many-valued function $\log (z)$ is only locally feasible, it can take place only in terms of local sections of the exponential covering $\exp (z)$. More precisely, each section defined on $\mathbb{C}-\{$ ray $\}$ constitutes an inversion of $\exp (z)$ only locally, and thus contributes, to a local determination of the logarithm. Each section bears the form $\log (z)+2 \kappa i \pi$, where $\kappa$ is an integer. The compatible gluing of all these local determinations over their non-trivial overlaps gives rise to the Riemann surface of the many-valued function $\log (z)$, as follows: 


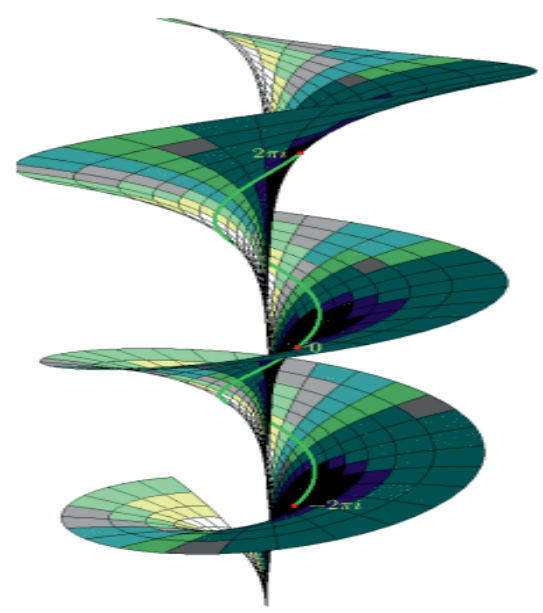

The multiply-extended aspect of a many-valued function of one complex variable lead naturally to the first topological considerations regarding the connectivity of surfaces. In particular, a piece of a surface is connected when any two points in it can be joined by a path. Riemann's method of probing the connectivity of surfaces is based on the notion of boundary cuts, which are simple paths joining two points on the boundary. A surface is simply connected if any boundary cut dissects it into two simply connected pieces rendering it disconnected.

In general, the type of connectivity of a surface involves the counting of the number of boundary cuts and the number of simply connected pieces obtained after performing these cuts. The basic idea is that if a surface is cut into $m$ simply connected pieces by $n$ boundary cuts, then the number $(m-n)$, for $m$ and $n$ variable, remains constant. According to Riemann, this constant captures the order of connectivity of the surface.

With this stipulation, a connected surface is judged to be $n$-fold connected if a system of $(n-1)$ boundary cuts are required to make it simply connected. Of course, these considerations pertain to a surface with boundary. In order that they become applicable to a closed surface it is necessary the metamorphosis of this surface to another one with a boundary. In particular, if we make a puncture at any point of a closed surface, then the analysis takes place by considering a cross section starting from this point and returning to it, giving rise to a loop, or closed curve.

Thus, whenever it is possible to draw $n$ closed curves $\alpha_{1}, \alpha_{2}$, $\ldots, \alpha_{n}$ on a surface $T$, which, either taken separately, or taken together, do not form the complete boundary of part of the surface, but 
which, joined to any other closed curve, do form the boundary of part of the surface, then the surface is. $(n+1)$-ply connected.

The next fundamental notion refers to the topological genus of a closed surface, especially concerning its relation with the connectivity order of a closed surface. If $2 p$ is the maximum number of closed curves (being allowed to intersect) which may be drawn on a closed surface $T$ without rendering it disconnected, then $p$ is the genus of this surface.

The genus is characterized as a complete homeomorphism invariant, meaning that two (compact and orientable) surfaces without boundary are homeomorphic, if and only if they have the same genus. Moreover, $2 p$ is identified as the rank of the first homology group of $T$, i.e. its first Betti number. Finally, it is straightforward to obtain the relation of the connectivity order $c$ of a closed surface $T$ with the genus $p$ of this surface, namely $c=2 p+1$.

According to William Kingdon Clifford, a closed curve on a surface should be thought of as a circuit. If it is possible to move a circuit continuously on the surface until it shrinks up into a point, the circuit is called reducible; otherwise it is irreducible. In general, there is a finite number of irreducible circuits on a closed surface which are independent. An independent circuit is characterized as one that by continuous motion cannot be made to coincide with a curve made out of the others.

In particular, for a surface bearing $p$ holes there are $2 p$ independent irreducible circuits; one around each hole, and one through each hole.

Conclusively, Riemann's metaphora addressing the ramification of a many-valued function, may be summarized as follows:

a eventuation of obstacles until each closed curve that embraces them plays the role of a boundary for a portion of the universal covering surface;

b application of boundary cuts;

c spreading out into all possible distinct covering branches; and,

d amalgamation of all the covering branches to obtain the simply-connected universal covering surface.

In the context of this metaphora, the relation between two different simply connected regions is expressed through Riemann's mapping theorem. More specifically, any two simply connected regions can be mapped conformally, that is, in an oriented angle-preserving way, onto one another. In particular, any such simply connected plane region can be mapped conformally onto the unit disc $\mathbb{D}$. 
The generalization of the Riemann mapping theorem to what is called the uniformization theorem for Riemann surfaces, proved by Henri Poincaré and Paul Koebe, states that any simply connected Riemann surface is isomorphic, meaning conformally equivalent in this context, to the Riemann sphere, to the complex plane, or to the unit disk. The term uniformization accords to the process of unfolding a multiply-extended variable magnitude to the universal covering, simply-connected, Riemann surface of this function, where it becomes uniformly valued. The type of uniformization applicable to a surface with holes depends on the genus of this surface. More precisely, those of genus 0 are their own universal covering Riemann surfaces, isomorphic to the Riemann sphere; those of genus 1 are universally covered by the complex plane; and all those of genus at least 2 are universally covered by the unit disk.

The basic idea here is the following: If we think of a hole on a surface as an obstacle, then the emergent simply-connected geometric unfolding of the great majority of these surfaces, involving all cases where we have two or more obstacles, conforms to the norms of neither the spherical, nor the flat geometry, but to those of the hyperbolic geometry.

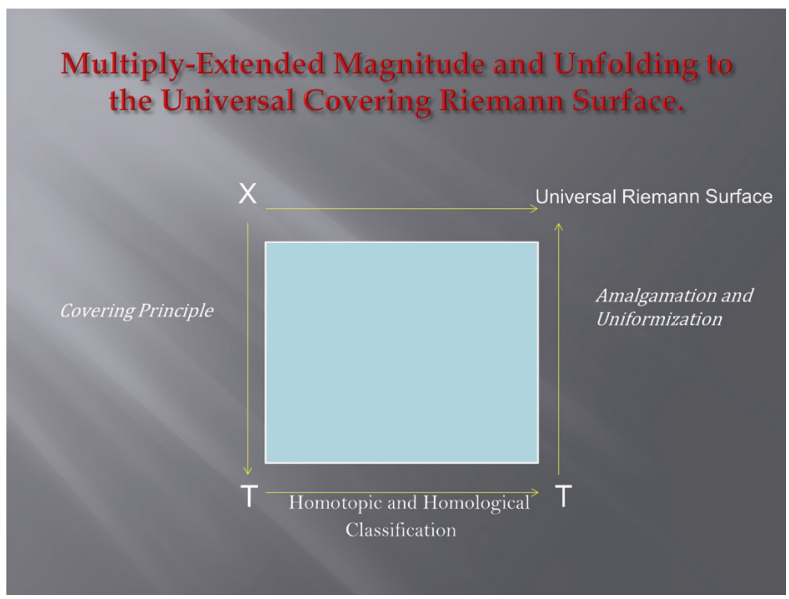

The hyperbolic plane bears four different models by means of which it can be explicated. The first is the upper half complex plane model, the second is the Beltrami-Klein projective disk model, the third is the Lorentz hyperboloid model, and the fourth is the Poincaré conformal 
unit disk model in the complex plane. We are going to focus on the conformal disk, and the upper half plane model, in what follows.

The points of the Poincaré disk model of the hyperbolic plane are the points which are interior to the unit disk in the complex plane, and the lines are the diameters and the arcs of circles which are orthogonal to the boundary of the disk. The definition of the distance between two points $z_{1}$ and $z_{2}$ in the interior of the disk is secured by constructing the hyperbolic line joining them. We also take into account that $\zeta_{1}$ and $\zeta_{2}$ are the endpoints of the diameter or arc of circle determining the hyperbolic line. Then the distance between the points $z_{1}$ and $z_{2}$ is:

$$
d\left(z_{1}, z_{2}\right)=\left|\log \left(z_{1}, z_{2}, \zeta_{1}, \zeta_{2}\right)\right|
$$

where $\left(z_{1}, z_{2}, \zeta_{1}, \zeta_{2}\right)$ denotes the cross-ratio of these four points, which is real and positive, so that the logarithm is definable. The geodesics in the unit disk are the circles and the lines in the plane that are orthogonal to the unit circle at two points.

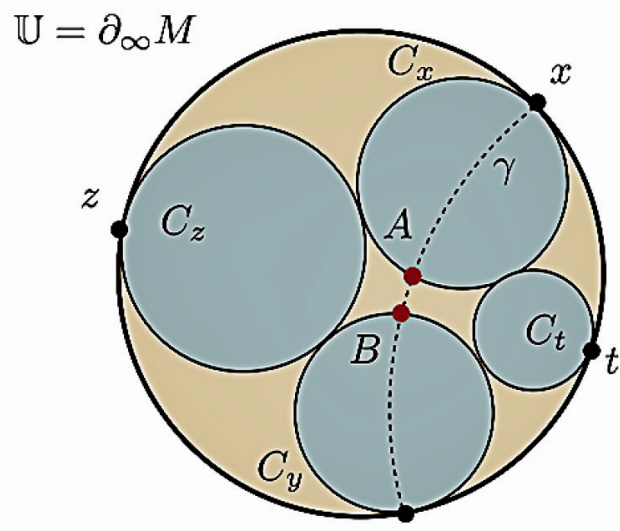

$y$

Poincaré assumes the existence of a world enclosed in a large boundary circle and subject to the following law: The temperature in this world is not uniform; it is largest at the center, and it diminishes as one moves away from the center, so that it reduces to absolute zero when one reaches the boundary circle where this world is enclosed. He considers the non-uniform variation of the temperature as follows: Let $R$ be the 
radius of the limit boundary circle, and let $r$ be the distance from the point under consideration to the centre of this circle. The absolute temperature will be proportional to $R^{2}-r^{2}$. He additionally assumes that, in this world, all bodies have the same coefficient of dilatation, in such a way that the length of any ruler shall be proportional to its absolute temperature. Finally, he assumes that an object transported from one point to another, whose temperature is different, shall immediately reach thermal equilibrium with its new location. A moving object will then become smaller and smaller as it approaches the boundary circle. If this world is finite from the point of view of our customary geometry, it will appear infinite to its inhabitants. In fact, when they intend to approach the boundary circle, they will get colder and become smaller and smaller. The steps they take are therefore also smaller and smaller, so that they can never actually reach this boundary.

In the same geometric context, Harold Scott Coxeter remarks that the conformal disk model is an inversive model of the infinite hyperbolic plane geometrically, using a circular "nutshell" figure to stand for the Absolute. When Hamlet exclaims (in Act II, Scene II) "I could be bounded in a nutshell and count myself a king of infinite space" he is providing a poetic anticipation of Poincaré's disk model.

Note that angles in the unit disk model of the hyperbolic plane are measured in the Euclidean way, so that the measure of the angle between two hyperbolic lines is the Euclidean measure of the angle between their tangents. Moreover, it is not adequate to consider only the hyperbolic plane disregarding its boundary. In the disk model, the boundary is realized by the unit circle.

Let us consider now the upper half plane model of the hyperbolic space. Here the points of the geometry are the points in the upper half plane, and the lines are either vertical rays from points on the real axis or semicircles with diameter on the real axis. Given two points $z_{1}$ and $z_{2}$ in the upper half plane, the Euclidean perpendicular bisector of the Euclidean segment joining them meets the real axis at the center of a semicircle through the two points.

If we consider that $\zeta_{1}$ and $\zeta_{2}$ are the endpoints of the semicircle, we can define the notion of distance in this model as in the Poincaré disk model, i.e. $d\left(z_{1}, z_{2}\right)=\left|\log \left(z_{1}, z_{2}, \zeta_{1}, \zeta_{2}\right)\right|$. Since an August Möbius transformation exists, which maps the unit disk model to the upper half plane model of the hyperbolic plane, there is a one-to-one mapping of one model to the other, which preserves all the hyperbolic distances and constitutes these two models of the hyperbolic plane as isometric images of one to the other. 


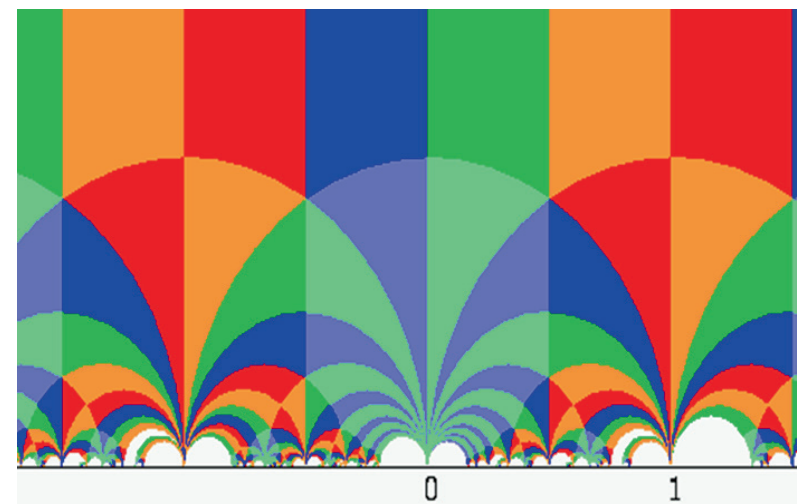

The Uniformization theorem encapsulates, via the notion of conformal equivalence, the geometry of the universal covering space, where the the complete unfolding of a multi-valued function takes place. In particular, since the universal covering is a simply-connected geometric space, the function becomes uniformly-valued on this space. From the algebraic-topological view of the first homotopy or, equivalently, fundamental group, Riemann's metaphora to the universal covering space of a base topological surface bearing holes targets the annihilation of the fundamental group of the base by continuous unfolding until trivialization.

Concomitantly, from an information-theoretic perspective, the same metahora may be thought of as a temporal process of complexity contraction, or symmetry reduction, in the Galois theoretic sense. More specifically, the monodromy action of the fundamental group on the fiber of the universal covering space over a marked point amounts to the Galois group-theoretic encoding of Riemann's covering principle utilizing Poincaré's first homotopy group as a Galois group.

According to the Uniformization theorem, the type of uniformization applicable to a topological surface bearing holes depends on the genus of this surface. Precisely, those of genus 0 constitute their own universal covering Riemann surfaces, which due to simple-connectivity, are all isomorphic to the Riemann sphere; those of genus 1 are universally covered by the complex plane; and those of genus at least 2 are universally covered by the unit disk.

Poincarés conception of uniformization is enacted in terms of a metaphora from the topological and complex analytic domain to the geometric domain. In the latter domain only three types of geometric spaces exist distinguishable by their curvature; the three classical geometries of constant curvature. Namely, the Riemann sphere bearing 
positive curvature, the Euclidean (or complex) plane bearing zero curvature, and the hyperbolic plane bearing negative curvature. Analogously to the case of elliptic functions, i.e. doubly-periodic functions, becoming uniformly valued on the complex plane, another type of functions, becoming uniformly valued on the hyperbolic plane, exist. These functions are called Fuchsian functions, since they have been first introduced by Lazarus Immanuel Fuchs in relation to the solution of differential equations with singular points. In this manner, the Fuchsian functions are to the geometry of the hyperbolic plane what the doubly-periodic functions are to the geometry of the Euclidean plane.

The metaphora from the geometric to the complex-analytic and topological domain emerges through the action of a certain group of symmetries or, automorphisms, on one of these three classical geometries of constant curvature. Geometrically, the group of symmetries is an isometry group of transformations that induces a tessellation on the simply-connected universal covering space, identified with one of the three types. In particular, tessellation implies the existence of a fundamental polygon that periodically tiles the universal covering space. In the case of doubly-periodic functions, the fundamental polygon is a parallelogram that tiles the complex plane, thus analogously, in the case of Fuchsian functions the fundamental polygon always possesses more than four sides tiling the hyperbolic plane.

The metaphora from geometry to analysis emerges by the consideration of this group as a group of conformal symmetries. In the case of the hyperbolic plane, this group is called a Fuchsian group. Finally, the metaphora from geometry to topology emerges by the consideration of this group in terms of the fundamental group of the underlying topological surface bearing holes, and which is universally covered by the corresponding tessellated geometric space. Concomitantly, a metaphora also emerges from geometry to algebra by identifying the same group of symmetries as the Galois group of covering automorphisms of the universal covering space. In this way, every Riemann surface can be realized as the topological quotient with respect to the action of a certain group of conformal symmetries on the three types of geometric spaces.

Concerning all of these three types, the conformal symmetries are Möbius transformations, i.e. fractional linear transformations, or else, homographies. In more detail, the only group acting on the sphere is the trivial group, so the only Riemann surface that we obtain is the sphere itself with the conformal structure. For the complex plane, the group of conformal symmetries is either cyclic, or a group generated by two independent translations on the complex plane, which captures the elliptic functions. Therefore, the only surfaces that can emerge by taking the quotient are the complex plane itself, considered as equivalent to the sphere bearing one puncture, the sphere bearing two punctures, and the 
torus. Every other Riemann surface is actually the quotient of the hyperbolic plane by a group of conformal symmetries of the hyperbolic plane. Such a group, a Fuchsian group, must be discrete and should act properly discontinuously.

The important idea is that, since a Fuchsian group is also a group of isometries of the hyperbolic geometric plane, the geometric structure descends under the quotient morphism to the Riemann surface. Due to this property, every Riemann surface comes equipped with a natural intrinsic geometry, completing the metaphora from geometry to complex analysis. Note that in the generic case, this geometry is non-Euclidean, namely it is of the hyperbolic type. Moreover, since the action of a Fuchsian group, as a discrete group of conformal isometries, does not have any fixed points, the Fuchsian group is isomorphic with the fundamental group of the Riemann surface, so completing the metaphora from geometry to topology as well.

We conclude that, essentially every Riemann surface, with the exception of the sphere, the plane, the punctured plane, and the torus, arise as quotients of the hyperbolic plane by a discrete and properly discontinuous group of conformal isometries, inheriting its own characteristic hyperbolic geometry by projection in this way. Conversely, the hyperbolic plane, instantiates the universal covering space of all those Riemann surfaces arising in the above manner, whose Galois group of covering automorphisms is identical with the fundamental group of the Riemann surface it covers universally, being in turn, identical with the pertinent Fuchsian group of conformal isometries.

Let us consider the subgroup of Möbius transformations, defined on the Riemann sphere, that map the unit disk into itself. The composition of any Möbius transformation in this subgroup with complex conjugation also maps the unit disk onto itself. Thus, we may consider the subgroup $I$ of the group of extended Möbius transformations (including antihomographies) mapping the unit disk onto itself. Since automorphisms in the unit disk belonging to this subgroup map lines and circles to lines and circles and are also conformal, the hyperbolic line between any two points in the interior of the unit disk will be mapped to a hyperbolic line between their respective images. Moreover, since transformations in $I$ preserve the cross-ratio of four points on the same line or on the same circle, they preserve the hyperbolic distance between two points in the unit disk. Therefore, the conformal automorphisms of the unit disk are isometries of the Poincaré disk model of the hyperbolic plane, and thus, $I$ is the isometry group of the Poincaré disk model of the hyperbolic plane. Notice that the action of the isometry group $I$ on the open unit disk is a transitive group action. 
Restricting our focus only on Möbius transformations, that is, on homographies, these symmetries of the extended real line preserving the upper half plane are characterized as linear fractional transformations with real parameters $a, b, c$, and $d$, where $a b-c d>0$. This group is isomorphic to the projective group $\operatorname{PSL}(2, \mathbb{R})$, the group of $2 \times 2$ matrices with real coefficients, whose determinant is equal to 1 , and each matrix $M$ is considered as equivalent to $-M$. Additionally, it follows that, each of these Möbius transformations is an isometry of the upper half plane with its hyperbolic metric that preserves the orientation as well. In this model of the hyperbolic plane, the geodesics are the semicircles orthogonal to the real line including the vertical semilines.

Coming back to the Poincare disk model of the hyperbolic plane, a group of conformal isometries $I$ of the disk is a Fuchsian group if it is equipped with the discrete topology. Let us consider the action of a Fuchsian group on the hyperbolic disk. We say that a subset of the disk is a fundamental set for the Fuchsian group, if this subset contains exactly one point from every orbit or fiber of the Fuchsian group. The idea is to obtain a fundamental domain, an open subset of the disk contained in the closure of a fundamental set, which under the action of the Fuchsian group is able to tessellate the hyperbolic plane. It is easy to see that a connected convex hyperbolic polygon plays the role of a fundamental domain for the action of a Fuchsian group on the disk. Hence, for any given Fuchsian group there is a corresponding convex hyperbolic polygon that tessellates the disk. The Poincaré polygon theorem tackles the inverse issue, i.e. given a convex hyperbolic polygon, what are the conditions ensuring the existence of a Fuchsian group for which this polygon functions as a fundamental domain of its action?

For a hyperbolic polygon $P$ a side pairing of $P$ is an injective morphism from the set of sides of $P$ to the group of all isometries of the hyperbolic disk, such that for sides $s, s^{\prime}, g_{s}$ is an isometry with $g_{s}(s)=s^{\prime}, g_{s^{\prime}}=g_{s}{ }^{-1}$, and $g_{s}(P)$ has an empty intersection with $P$ for all sides $s$. Then the Poincaré polygon theorem states that considering $P$ a compact and connected hyperbolic polygon of the disk with a side pairing that generates a Fuchsian group of isometries, if every angle of $P$ is equal to $2 \pi / n$, for some $n \in \mathbb{N}$, then $P$ is a fundamental polygon for this Fuchsian group.

The process of relating the symmetries of tessellations of the hyperbolic disk to the annihilation of the fundamental group of the underlying Riemann surface is based on the conception of the hyperbolic disk that is being tessellated as the simply connected universal covering surface of this Riemann surface. The latter emerges by taking a quotient 
topology of the hyperbolic disk based on a specific fundamental domain hyperbolic polygon together with the procedure of side pairing. Thereby, the tessellated hyperbolic disk is the universal covering space of that particular quotient surface and the set of all covering transformations becomes isomorphic to the Fuchsian group that induces this tessellation. It is thus, also isomorphic to the fundamental group of the quotient space, the underlying Riemann surface.

In sum, a Fuchsian group represents a single Riemann surface, and inversely, every Riemann surface (modulo the exception of the cases mentioned in the beginning) is uniformizable by a concrete Fuchsian group acting on the hyperbolic disk up to conformal equivalence. Henceforth, all Riemann surfaces of topological genus greater than or equal to two can be identified with a quotient of the hyperbolic disk by a discrete group of conformal isometries. The precise connection between the conformal and the hyperbolic metric structure is expressed by the Schwarz-Pick theorem, asserting that every conformal automorphism of the hyperbolic disk is contracting for the hyperbolic metric.

The important notion characterizing Poincaré's metaphora in this setting is that a Fuchsian group used to set up a certain tessellation of the hyperbolic disk by hyperbolic polygons is isomorphic to the fundamental group of the quotient Riemann surface made with that same polygon by isometric side pairings.

This culminates in the threefold isomorphic manifestation of the same uniformizing group, comprehending simultaneously, the isometry group inducing a tessellation on the hyperbolic disk, the group of covering transformations of the universal covering space identified with the tessellated hyperbolic disk, and the fundamental group of the underlying quotient Riemann surface. The unifying power of this threefold isomorphic manifestation of the group concept in relation to unveiling the nature of a Riemann surface is illuminated by the realization that the first of these manifestations is of geometric type (in particular, it pertains to the tessellation of the hyperbolic disk by isometries), the second is of the Galois type in a topological setting, and the third is of a homotopic type.

For example, we consider a regular hyperbolic octagon $O$, which is centered in the hyperbolic disk $\mathbb{H}$. We notice that a regular octagon in the Euclidean space has each angle equal to $6 \pi / 8$, thus its hyperbolic analogue must be less than that. In particular, since $O$ has eight sides, the Poincaré polygon theorem dictates that considering $O$ to be a compact and connected hyperbolic polygon of the disk with a side pairing that generates a Fuchsian group of isometries, every angle of $O$ must be equal to $2 \pi / 8$, where $O$ is a fundamental polygon for this Fuchsian group, denoted by $F$. Since the action of $F$ must be free and properly discontinuous, after fixing one vertex of $O$ there can be 
exactly 8 distinct elements of $F$ that map each vertex of $O$ to the fixed vertex.

Equivalently, this means that a Fuchsian group $F$ that has $O$ as its fundamental domain polygon, can be identified as a group of covering automorphisms of the universal covering space identified with the tessellated hyperbolic disk by hyperbolic octagons. Such a Fuchsian group $F$ is realized by isometric side pairings of $O$, and is cast isomorphic to the fundamental group of the underlying Riemann surface, obtained by the quotient $\mathbb{H} \rightarrow \mathbb{H} / F$. The latter is being covered universally by the octagonally tessellated hyperbolic disk, and is identified as a genus two surface $\Sigma_{2}$, isomorphic with the double torus.

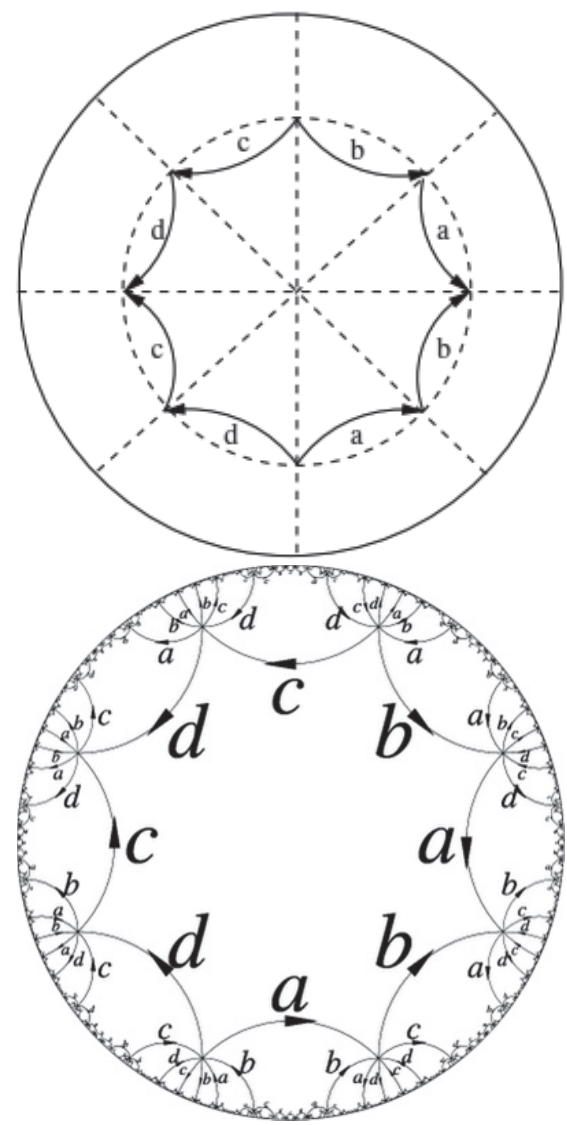



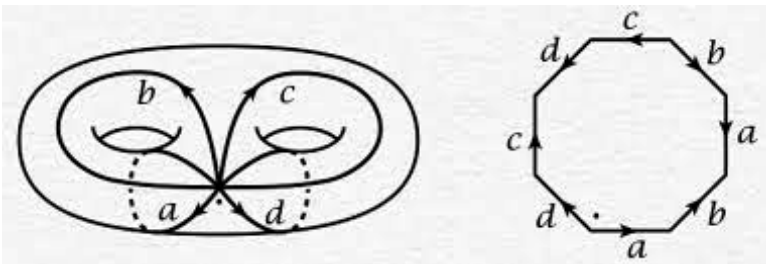

Since $F$ is realized by isometric side pairings of $O$, we have a generator for each pair of sides, i.e. we have 4 generators, denoted by $a, b, c, d$. Accordingly, the underlying Riemann surface $\Sigma_{2}$ is manifested in terms of the hyperbolic octagon $O$ with sides identified in pairs according to the boundary relation $[a, b] \cdot[c, d]=1$ under the action of $F$. Thus, in turn, the Fuchsian group $F$ that has $O$ as its fundamental domain, is cast isomorphic with the fundamental group of $\Sigma_{2}$, i.e. the group generated freely by the 4 generators $a, b, c, d$ subject to the boundary relation $[a, b] \cdot[c, d]=1$. OF A BOUQUET

The abstract algebraic notion of a group is amenable to an elucidating metaphora that crosses from algebra to geometry by the encoding bridge constituted by the action of a group. In the simplest case of a group action on a set, if $S_{X}$ denotes the symmetric group of a set $X$, i.e. the group of permutations of the set $X$, then the action of a group $G$ on $X$ is equivalent to a group homomorphism from $G$ to $S_{X}$, denoted by $G \rightarrow S_{X}$.

From this correlation, each element of $G$ gives rise to a permutation of $X$ by acting upon it, in such a way that the composition and the identities are preserved in both groups. The permutation group $S_{X}$ should be thought of geometrically as the group of symmetries of $X$, thus a group action of $G$ on $X$ provides the means of realization of $X$ in terms of the group of symmetries of $X$. If no fixed point emerges under the action of $G$ on $X$, we say that this action is free. Additionally, if we consider the orbit or fiber of the action of $G$ on $X$ at $x$, i.e. the set $G \cdot x$, and this set reproduces the whole set $X$ under the action of $G$, then this action is characterized as transitive. 
Most important is the fact that a group may act on itself, and this can happen in two ways. First, a group may act on itself by left multiplication, defined by $g \cdot h=g h$. Second, a group may act on itself by conjugation, defined by $g \diamond h=g h g^{-1}$. We recall that the second action distinguishes the normal subgroups of a group as well, as those that remain invariant under conjugation. Only the first action is free and transitive, in general. Since, the self-action by left multiplication is realized as a group homomorphism $G \rightarrow S_{G}$, every group is realized isomorphically as a subgroup of some symmetric group.

The above metaphora from algebra to geometry is based on the idea of realization of a group in terms of its action on a set, including the cases of self-action. The issue is whether or not we can identify another geometric realization of a group that is more intrinsic, in the sense that it is capable of geometrizing the notion of a group itself. For this purpose, we have to consider a group not in terms of its action on a set, but in terms of its action on a graph, called the Arthur Cayley graph of this group. This task is enunciated by adopting the combinatorial perspective on group theory, which refers to the characterization of a group in terms of generators and relations, or equivalently, in terms of a presentation of the group. In the combinatorial setting, there appears first of all, the fundamental notion of a free group.

A free group is characterized by a certain number of generators without any additional defining relations beyond the existence of an inverse for each generator, according to the general requirements of the notion of a group. Therefore, every morphism of a set of free generators onto a set of elements of any group, is tantamount to a homomorphism of the free group into this group. Consequently, a presentation of a finitely generated group can be expressed as a quotient group of a certain free group of finite rank with respect to the congruence relations defined among the generators.

We consider that $S$ is a generating set of a group $G$. Then the Cayley graph of $G$ with respect to the generating set $S$ is a directed, labelled, or even colored, graph $\Gamma(G, S)$ whose set of vertices is the set of elements of $G$, such that there exists a directed edge from $g$ to $g s$ for every $g$ in $G$ and $s$ in $S$, labelled by $s$. Hence, if $s_{1}, S_{2}, \ldots$, $S_{n}$ is an ordered sequence of labels on an edge path in $\Gamma(G, S)$ from the identity 1 to $g$, then $g=s_{1} s_{2} \ldots s_{n}$. Conversely, if $g=s_{1} s_{2} \ldots s_{n}$, then an edge path on $\Gamma(G, S)$ from the identity 1 to $g$ exists, whose labels or colors are $s_{1}, s_{2}, \ldots, s_{n}$ in this order. Thus, we obtain a correspondence between edge paths in the Cayley graph from the 
identity 1 to $g$ and words in the generators representing $g$ in this manner.

Each element of a group $G$ induces a symmetry, i.e. an automorphism of the Cayley graph $\Gamma(G, S)$ of $G$ with respect to the generating set $S$ in the following way: The automorphism $Y_{g}$ associated with the element $g$ in $G$ is defined on the vertices of the Cayley graph by $Y_{g}(v)=g v$, identified with the left action of $G$ on itself. Since there exists at most one directed edge connecting any two vertices, and since no edges connect a vertex to itself, there can be one and only one way to extend this action to an automorphism of the entire directed, labelled (colored) graph, i.e. $Y_{g}$ sends the edge $v \rightarrow v s$ to the edge $g v \rightarrow g v s$. Further, considering that the word length of an element $g$ in $G$ with respect to the generating set $S$ is the length of the shortest word in $S \bigcup S^{-1}$ that is equal to $g$, we derive that the group morphism $G \rightarrow A u t \Gamma(G, S)$ is an isomorphism. In this combinatorial setting, the notion of word length bears a geometric signification, in that the word length of $g$ is the minimum number of edges in an edge path from the identity 1 to $g$.

The notion of the free group on two non-commutative generators bears a fundamental role. The simplest way to describe a free group is the following: We consider the set of elements $S=\left\{x_{1}, x_{2}, \ldots x_{n}\right\}$ in a group $G$. A word or ordered string $w \in\left\{S \cup S^{-1}\right\}^{\leftleftarrows}$ is said to be freely reduced if it does not contain a substring consisting of an element adjacent to its formal inverse. For instance, the ordered string $w=x y x^{-1} y^{-1}=[x, y]$ is freely reduced, while $z=x y^{-1} y x y$ is not. The group $G$ is a non-Abelian or, equivalently, a non-commutative free group with basis $S$ if $S$ is a set of generators for $G$ and no freely reduced string in the $x_{i}$ nor their inverses represents the identity of the group. The rank of a free group with basis $S$ is the number of elements of $S$. We denote a free group of rank 2 by $\Theta_{2}$. It can be easily shown that all free groups of the same rank are isomorphic replicas of each other. So we may identify all of them and talk universally of the non-Abelian free group on two generators $\Theta_{2}$.

The objective is to characterize the non-Abelian free group on two generators $\Theta_{2}$ as a group of symmetries, expressed as automorphisms of its Cayley graph with respect to any generating set. 
For this, we may consider $a$ and $b$ as the standard non-commuting generators of $\Theta_{2}$. If we start with the vertex corresponding to the identity 1 in $\Theta_{2}$, the empty word, then we have to consider four directed edges containing 1 , namely the edges connecting 1 with the vertices $a, b, a^{-1}$, and $b^{-1}$, exhausting in this way all four different elements of $\Theta_{2}$ with word length 1 . For each one of these four acquired vertices, there are going to be three new vertices connected to it, or equivalently, three new edges incident to it, according to the following:

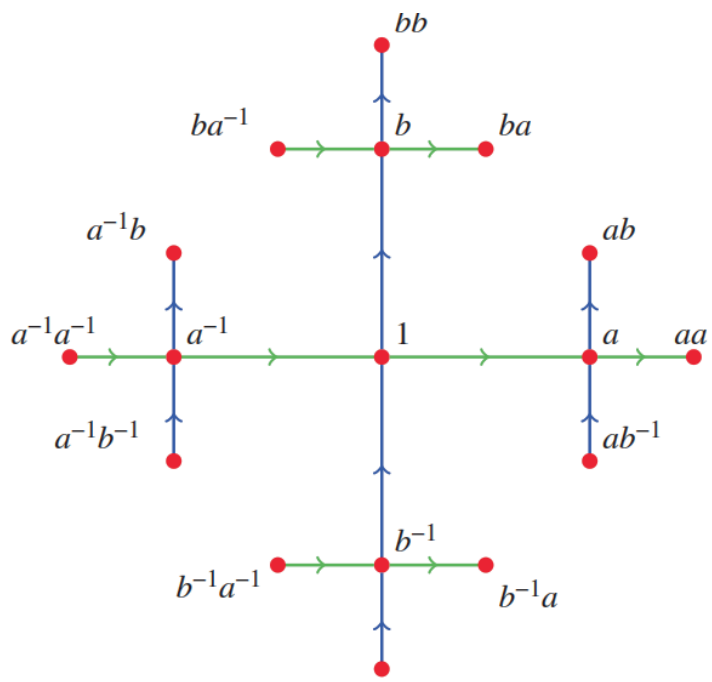

Notice that each new vertex is a reduced word on the generators $a$ and $b$, and moreover, since distinct reduced words provide distinct elements of $\Theta_{2}$, there is no vertex that emerges as new more than once in this procedure. Hence, the Cayley graph of the free group $\Theta_{2}$ with respect to any generating set is actually a tree. This is precisely the qualification of a Cayley graph characteristic of freeness in two non-commuting generators.

In more detail, the Cayley graph of the free group $\Theta_{2}$ is a tree whose vertices have valence four. From the perspective of this metaphora from algebra to geometry, the free group on two non-commuting generators $\Theta_{2}$ is characterized as the symmetry group of a 4-valent tree. A 4-valent tree is simply connected topologically, since there are no cycles, and can be also endowed with a metric making 
it into a geometric space. The considered metric is the path metric, i.e. the metric imposed on the set of its vertices such that the distance between two vertices is the length of the shortest path made through edges connecting these two vertices. Thus, we conclude that a group, together with a generating set, gives rise to a metric space, in such a way that its actions take place through isometries.

The action of the free group $\Theta_{2}$ on its Cayley graph, on the corresponding 4-valent tree, is an action without any fixed points, meaning that it is a free group action. It turns out that this property characterizes a group uniquely as a free group. Equivalently, if a group acts freely on a tree, then this group is a free group. Therefore, the qualification that a group is free amounts to the condition that this group acts freely on a tree, and in sum can completely characterized by its free action as a group on a tree. The consequence of this equivalence is called the Nielsen-Schreier theorem, stating that any subgroup of a free group is also free.

We recall now that the free group in two non-commuting generators has been utilized for the articulation of a temporal bond in terms of a Tripodal link, where the generators of this group are identified with two temporal actions whose composition is irreversible, and thus, non-commutative.

We conclude that, if the group $\Theta_{2}$, i.e. the non-commutative group in two generators is enunciated in terms of its temporal actions, then it is characterized uniquely as the symmetry group of a 4-valent tree and conversely. In this sense, the 4-valent tree deciphers the universal form of joint unfolding taking place by means of all possible combinatorial compositions of these temporal actions. The 4-valent tree is a simply-connected geometric space, identified in this way, as the universal covering or unfolding space generated by two non-commuting temporal actions. We note that the growth of this 4 -valent tree is exponential and boundless. The fundamental role of the free group in two non-commuting generators rests on the fact that a free group in any number of generators bigger than two is actually included as a subgroup of $\Theta_{2}$.

In turn, the 4-valent tree, being simply connected and amenable to the metric structure of its natural path metric, qualifies as a geometric space whose group of symmetries is $\Theta_{2}$. Taking into account that the action of $\Theta_{2}$ on this 4 -valent tree is free and transitive, it qualifies as a Galois action, which in turn, means that $\Theta_{2}$ is manifested as a group of covering automorphisms of this tree in its role as a universal covering 
space that annihilates the fundamental group of its quotient by this action.

We can elucidate further by considering the free group $\Theta_{2}$ in its function as the fundamental group of a bouquet of two unlinked circles (equivalently called a 2-rose, or a rose with two petals) whose universal simply-connected geometric covering space is a 4-valent tree.

The fact is that the non-commutative free group in two generators $\Theta_{2}$, expresses a genuine and non-reducible type of non-commutativity. To grasp this, it is indispensable to stress the behavior of the group commutator. The group-theoretic commutator induced by the generators of $\Theta_{2}$ :

$$
\left[\alpha, \beta^{-1}\right]=\alpha \beta^{-1} \alpha^{-1} \beta
$$

produces an irreducible non-commutative and ordered string of symbols in $\Theta_{2}$. This string represents a based loop $\gamma$ as a product loop, which is composed by the ordered composition of the based oriented loops $\alpha \circ \beta^{-1} \circ \alpha^{-1} \circ \beta$. The crucial observation is that deletion of both symbols $\alpha$ and $\alpha^{-1}$, reduces the group commutator to the identity 1. Clearly, the same behavior is encountered symmetrically for both $\beta$ and $\beta^{-1}$.

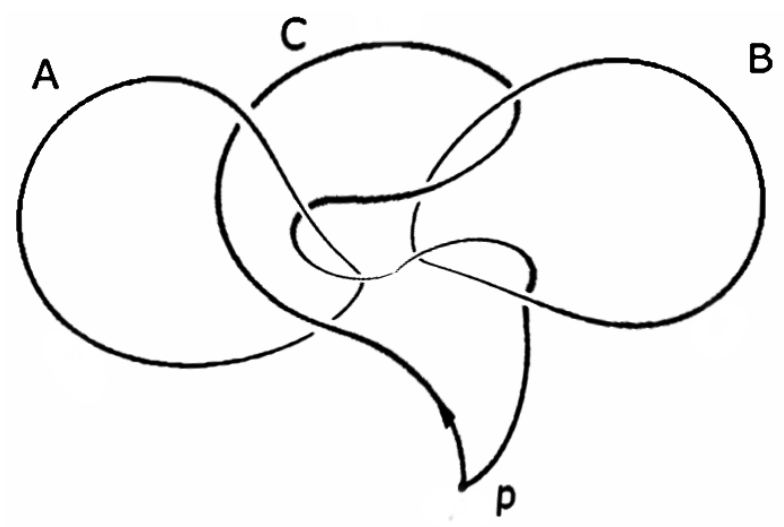

In particular, the group-theoretic commutator $\left[\alpha, \beta^{-1}\right]$ in $\Theta_{2}$, algebraically encodes the modular gluing condition of the based oriented loops $\alpha$ and $\beta^{-1}$, which in three dimensions corresponds to the 
Tripodal link. Because of this, the commutator $\left[\alpha, \beta^{-1}\right]$ constitutes the basic element of the fundamental group of a bouquet of two circles, identified with the group $\Theta_{2}$, from which the non-commutativity of this group is based on. The annihilation of this fundamental group takes place by means of the universal simply connected geometric covering space of the bouquet, identified as a 4-valent tree, whose Galois group of covering automorphisms is precisely $\Theta_{2}$.

We have deduced that the non-commutative ordered product $\gamma=\left[\alpha, \beta^{-1}\right]=\alpha \beta^{-1} \alpha^{-1} \beta$ is not contractible to the identity, due to the homotopic non-deformability of the commutator product loop to a trivial loop. Equivalently, $\gamma$ belongs to the non-trivial homotopy class of the fundamental group defined on the complement of two disjoint, or directly unlinked, topological circles $A$ and $B$. Conversely, we realize that $\gamma$ is actually reducible to the identity if $\alpha$ commutes with $\beta^{-1}$. Hence, the vanishing of the commutator $\gamma$ amounts to the Abelianization of the fundamental group, which in turn is identified with the Abelian first homology group.

The above provides a crucial insight into the working of Hurewicz's theorem, regarding the interrelation between the fundamental group and the first homology group in algebraic topology. According to Witold Hurewicz, the concept of homotopy is a mathematical formulation of the intuitive idea of a continuous transition between two geometrical configurations, whereas the concept of homology gives a mathematical precision to the intuitive idea of a curve bounding an "area", or a surface bounding a "volume". The crucial idea is that the basic process of homology theory consisting in decomposing a space into smaller pieces with simpler homology structure has no counterpart in homotopy theory.

In the preceding setting, the first homology group arises as the Abelian quotient of the fundamental group with respect to its normal subgroup generated by all commutators. In this way, reciprocally, non-trivial commutator elements in the fundamental group give a measure of deviation from its Abelian shadow manifested by its first homology group. Essentially, the based loops $\alpha, \beta$ in the fundamental group are viewed as oriented and commuting 1-cycles representing homology classes, and as such generate the first homology group by means of defining a canonical free $\mathbb{Z}$-module basis. 
Every Riemann surface corresponds to the action of a unique discontinuous group of conformal transformations on the Riemann sphere. More precisely, this is a claim regarding the issue of uniformization of Riemann surfaces by means of a free, transitive, and discontinuous group action. We have already seen that that a Fuchsian group can be used to set up a certain tessellation of the hyperbolic disk by hyperbolic polygons, where this group is isomorphic with the fundamental group of the quotient Riemann surface emerging from this polygon by isometric side pairings. In this sense, the conformal symmetries effected by a discontinuous group action are actually isometries of the hyperbolic disk.

Of especial interest here is the case of a figure bounded by a certain number of non-intersecting circles on the Riemann sphere, attention to which was first drawn by Friedrich Hermann Schottky, and then by Christian Felix Klein, concerning the new light it throws on matters of uniformization.

We recall that homographies or, Möbius transformations, are the conformal symmetries of patterns on the Riemann sphere. More specifically, Möbius transformations are classified into three types as loxodromic, parabolic, or elliptic. Loxodromic transformations have two fixed points, one of which may be physically thought of as attracting whereas the other one as repelling, and are conjugate to scaling by complex numbers except for scaling by unit complex numbers. These ones, whose multiplier is a positive real number, are also called hyperbolic transformations. Parabolic transformations have one fixed point and are conjugate to parallel translations. Elliptic transformations have two fixed points and are conjugate to rotations.

Since Möbius transformations are the symmetries of patterns on the Riemann sphere, we are interested on the type of pattern obtained, characterized as simultaneously symmetrical under the action of two non-commuting loxodromic Möbius transformations. The initial set-up consists of a single loxodromic transformation $A$ and a pair of non-overlapping disks $D$ and $D^{\prime}$, selected by the criterion that $A$ maps the outside of $D$ to the inside of $D^{\prime}$.

In this setting, we say that $A$ effects a pairing between $D$ and $D^{\prime}$. Since, the Möbius transformation $A$ is loxodromic, we consider that the repelling fixed point is inside $D$, and that the attracting fixed point of the transformation is inside $D^{\prime}$. We denote the inverse of $A$ by $A^{-1}:=a$. In this manner, we apply the notational convention that the outside of the disk $D_{A}$ is mapped by $a$ to the inside of the disk 
$D_{a}$, and the inside of the disk $D_{A}$ is mapped to the outside of the disk $D_{a}$. In the same way, the bounding circle $C_{A}$ of the disk $D_{A}$ is mapped by $a$ to the bounding circle $C_{a}$ of the disk $D_{a}$; whence the attracting fixed point of $a, F^{+}(a)$, is inside $D_{a}$, and the repelling fixed point of $a, F^{-}(a)$, is inside $D_{A}$.

We note that successive images of $D_{A}$ and $D_{a}$ under respective iterative loxodromic actions are nesting down toward the attractive and the repelling fixed points of these actions. More concretely, iterative powers of $a$ contract the disk $D_{a}$ to smaller and smaller disks containing $F^{+}(a)$, whereas iterative powers of $a^{-1}=A$ contract the disk $D_{A}$ to smaller and smaller disks containing $F^{-}(a)$.

At the next stage, we consider two non-commuting loxodromic transformations $a$ and $b$ which act jointly on a constellation of four non-overlapping disks, denoted by $D_{a}, D_{b}, D_{A}, D_{B}$, where $a^{-1}=A$ and $b^{-1}=B$, according to the generalization of the preceding. Note, that since Möbius transformations always map circles to circles, when any of the four transformations $a, b, A, B$ is applied to any of the four respective disks, all four images are disks themselves. Thus, the transformation a maps the outside of $D_{A}$ to the inside of $D_{a}$, and the inside of $D_{A}$ absorbs everything except $D_{a}$, i.e. $a\left(D_{A}\right)=D_{A} \cup D_{B} \cup D_{b} \cup O$, where $O$ is the region outside the four disks.

Analogous considerations hold for the action of the loxodromic $b$ and its inverse. It is clear that the two non-commuting loxodromic transformations $a$ and $b$ acting jointly on this constellation of four non-overlapping disks, and without imposing any further relations, generate a free non-commutative group, i.e. the group $\Theta_{2}$, called in this context the Schottky group on two generators. Repeated application of all the four transformations, i.e. applying $a$ and $b$ and their inverses to form words in the free group $\Theta_{2}$, leads to an action of this group on the constellation of four non-overlapping disks that is characterized by a repetitive pattern at all different levels of magnification.

More precisely, each of the disks involved contains three smaller disks, each of which in turn contains three smaller disks, and so on ad 
infinitum. As a result, disks within disks come to nest down leaving invariant at the limit a type of Cantorian dust, consisting of those points that belong to the disks at every single level of the process. We may think of a single particle of dust at the limiting end point of each infinite chain of disks nested within each other by the action of $\Theta_{2}$. In this sense, the limit-set of this group action consists of these particles of dust, and hence, this limit-set is the invariant pattern under the action of $\Theta_{2}$ in the Schottky setting identified with the closure of all attracting and repelling points.

\subsection{HYPERBOLIC BOUNDARY: INVARIANT PATTERN OF FREE GROUP'S ACTION}

We remind that the non-commutative group $\Theta_{2}$ is realized by its free action on a 4-valent tree, giving rise to a boundless exponential schema of growth. In turn, this 4 -valent tree is simply connected and amenable to the metric structure of its natural path metric. Thus, it qualifies as a geometric space whose group of symmetries is $\Theta_{2}$. Taking into account that the action of $\Theta_{2}$ on this 4 -valent tree is free and transitive, it qualifies as a Galois action.

Thereby, $\Theta_{2}$ is realized as a group of covering automorphisms of a 4-valent tree in the role of the latter as a universal covering space annihilating the fundamental group of its quotient by the action of $\Theta_{2}$. In the present setting, the action of $\Theta_{2}$ as a free group generated by two non-commuting loxodromic transformations $a$ and $b$ which act jointly on a constellation of four non-overlapping disks on the Riemann sphere may be comprehended by means of the concomitant 4-valent tree. Equivalently, this 4-valent tree is able to record the associated pattern of nesting disks within disks under the action of $\Theta_{2}$, by explicating graphically the organization pattern of nested disks at different levels of semantic unfolding.

If we consider $a$ and $b$ as the standard non-commuting generators of $\Theta_{2}$, and we start with the vertex corresponding to the identity 1 in $\Theta_{2}$ at level 0 , i.e. the empty word, then we have to consider four directed edges containing 1, i.e. the edges connecting 1 with the vertices $a, b, a^{-1}$, and $b^{-1}$, exhausting in this way all four different elements of $\Theta_{2}$ with word length 1 , at level 1 . In the present 
setting, we may identify these four vertices with the representation of the four disjoint disks $D_{a}, D_{b}, D_{A}, D_{B}$ respectively. For each one of these four acquired vertices, there are going to be three new vertices connected to it, or equivalently, three new edges incident to it at level 2 , according to the following diagram:

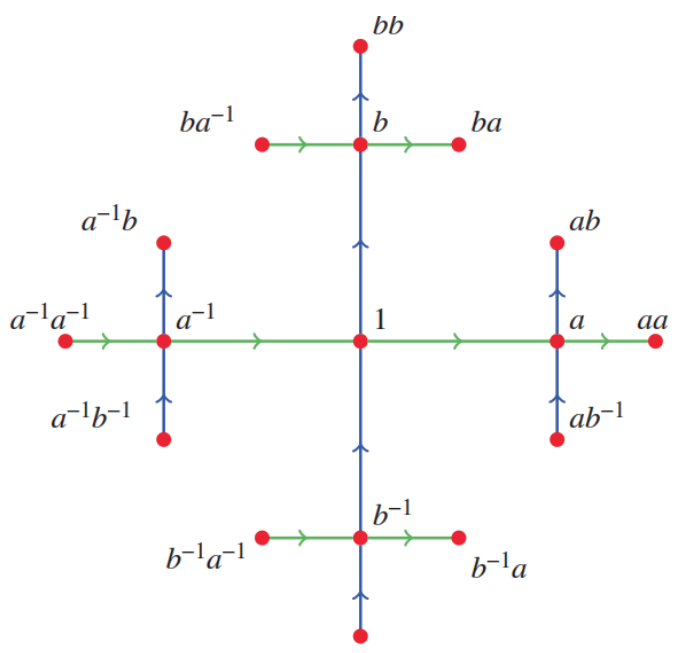

We note that the representation of each new disk is formed by the application of a certain reduced word in the group $\Theta_{2}$ to one of the initial four disjoint disks $D_{a}, D_{b}, D_{A}, D_{B}$. Moreover, the name of each new disk at a level remembers the original disk at the previous level from which it emamantes, together with the action applied to the latter, so the disk $D_{a a}$ at level 2 is tautologous to $a\left(D_{a}\right)$, and so on. Therefore, the unfolding of the 4-valent tree represents the nesting of disks, for instance the disk $D_{a b A}=a\left(D_{b A}\right)=a b\left(D_{A}\right)$ at level 3, is inside the disk $D_{a b}$ at level 2, which correspondingly is inside the disk $D_{a}$ at level 1. In consequence, we obtain a nested chain of disk inclusions $D_{a b A} \subset D_{a b} \subset D_{a}$. 


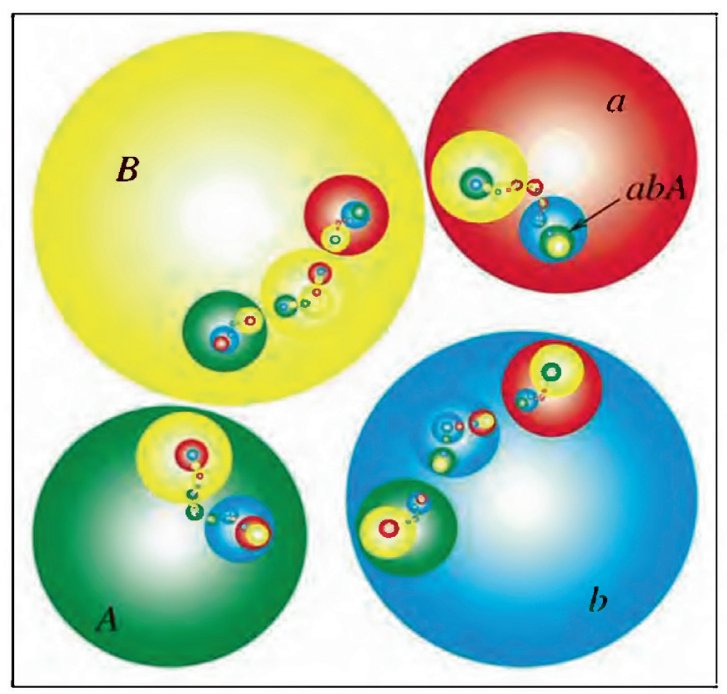

The limit-set of the action of $\Theta_{2}$ consisting of a type of Cantorian dust, and hence representing, the invariant pattern under the action of $\Theta_{2}$ identified with the closure of all attracting and repelling fixed points of loxodromic transformations on the four disjoint disks on the Riemann sphere, may be thought of as the boundary of the 4-valent representing tree. The region on the Riemann sphere, which is outside the initial four disjoint disks, denoted by $O$, is the fundamental domain of the group action of $\Theta_{2}$. The region of the Riemann sphere that is filled up by the copies of the fundamental domain $O$ under the action of $\Theta_{2}$ is called the domain of discontinuity of this group action because all replicas of $O$ remain separated from each other without getting stacked.

Each of the loxodromic transformations generators of $\Theta_{2}$, i.e. $a$, $b$, and their inverses $a^{-1}$, and $b^{-1}$, replicate conformally the fundamental domain $O$ within each of the four involved disjoint disks accordingly. By these means, under the action of $\Theta_{2}$ the fundamental domain $O$ tessellates the whole Riemann sphere except the limit-set, identified with the boundary of the infinite 4-valent tree of unfolding. 


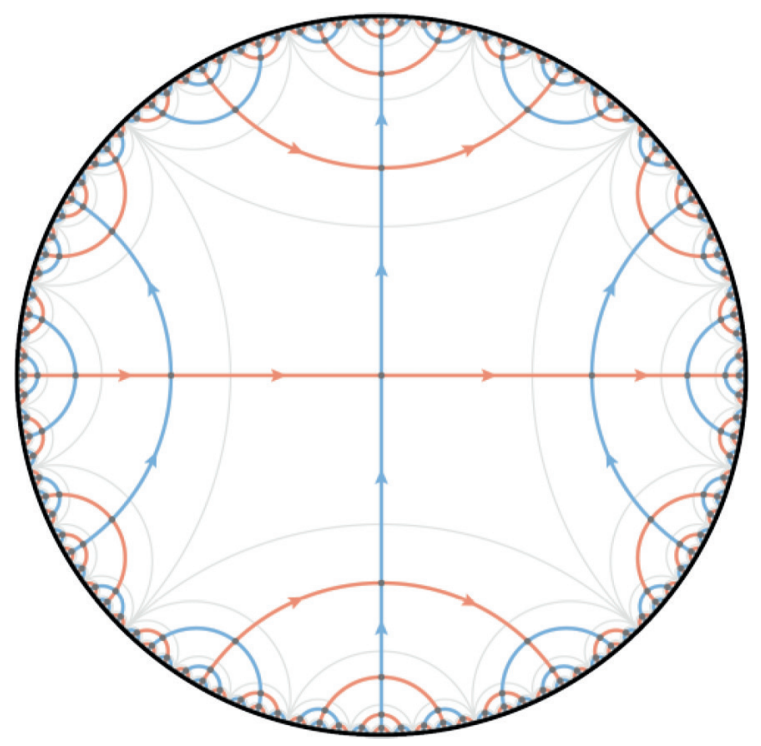

It was Klein's student Walther von Dyck, who first realized that Klein's emphasis on Schottky's model, involving a group acting on the Riemann sphere where no reduced nontrivial combination of elements fixes the sphere, corresponds to the action of a free group, providing this group with its name. In this context, what is called "Klein's criterion", furnishes the conditions under which a group acting on a set is characterized as a free one. "Klein's criterion" assumes that $a$ and $b$ generate a group acting on a set $S$. If $S$ has disjoint nonempty subsets $S_{a}$ and $S_{b}$, and $a^{n}\left(S_{b}\right) \subset S_{a}$ as well as $b^{n}\left(S_{a}\right) \subset S_{b}$, then this group is isomorphic to $\Theta_{2}$. Klein's criterion has been employed in the context of the group of Möbius transformations, i.e. the conformal automorphisms of the Riemann sphere, characterized as linear fractional transformations with complex coefficients $a, b, c$, and $d$, where $a b-c d=1$. This group is isomorphic to the projective group $\operatorname{PSL}(2, \mathbb{C})$, the group of $2 \times 2$ matrices with complex coefficients, whose determinant is equal to 1 , and each matrix $M$ is considered as equivalent to $-M$. Note also that the projective group $\operatorname{PSL}(2, \mathbb{C})$ is the group of metrical symmetries, i.e. orientation-preserving isometries of the three-dimensional hyperbolic space. Klein formulated his criterion in order to identify a Schottky group as a free subgroup generated by two loxodromic generators of the projective group $\operatorname{PSL}(2, \mathbb{C})$. 
A natural question arising in the context of Riemann surfaces is whether it is possible to consider the free non-commutative group on two generators as a uniformizing group in terms of its action on the fundamental domain $O$ and the induced tessellation of the Riemann sphere except the identified limit-set, i.e. the closure of the attracting and repelling fixed points of the two non-commuting loxodromic generators of this free group, identified as a Schottky group in the setting of our discussion. Notice that the free group on two generators is manifested here as a discrete subgroup of $\operatorname{PSL}(2, \mathbb{C})$.

This should be compared with the action of a Fuchsian group, i.e. a group of conformal isometries of the extended real line that preserve the upper half plane, identified as a discrete subgroup of $\operatorname{PSL}(2, \mathbb{R})$, and used to set up a certain tessellation of the hyperbolic disk by hyperbolic polygons. We emphasize that the same group is isomorphic to the fundamental group of the quotient Riemann surface made with that same polygon by isometric side pairings, culminating on the threefold isomorphic manifestation of the same uniformizing Fuchsian group: as an isometry group inducing a tessellation on the hyperbolic disk; as the group of covering transformations of the universal covering space identified with the tessellated hyperbolic disk; and as the fundamental group of the underlying quotient Riemann surface.

For the purpose of uniformization in the Schottky setting, we consider the region $O$ on the Riemann sphere being outside the four disjoint disks, or equivalently, the fundamental domain of the group action of $\Theta_{2}$, generated by $a, b$, where their inverses are $a^{-1}=A$, and $b^{-1}=B$. We may glue each point $p$ on the boundary circle $C_{a}$ of the missing disk $D_{a}$ with its symmetrical point $A(p)$ on the boundary circle $C_{A}$ of the missing disk $D_{A}$ under the action of the generator $a$ of $\Theta_{2}$. The same goes for the two other boundary circles $C_{b}$ and $C_{B}$ of the missing disks $D_{b}$ and $D_{B}$ respectively, under the action of the generator $b$. The first gluing gives rise to a handle on the Riemann sphere, and analogously the second gluing gives rise to another handle.

Thus, we obtain a Riemann surface bearing two handles, i.e. a Riemann surface of genus two, identified with the double torus. In this manner, the double torus admits uniformization by means of the action of the free group $\Theta_{2}$ on the Riemann sphere without four disjoint disks. Note that in this case, the action of the free group on two generators $\Theta_{2}$ 
is not considered on the simply connected universal covering space of the double torus, but on the covering space depicted by the domain of discontinuity of this free group action.

More generally, the retrosection theorem of Koebe, states that every compact Riemann surface $S$ can be represented as the quotient $\Omega / \Theta_{g}$, where $\Theta_{g}$ is the free group on $g$ generators, $g$ is the genus of the Riemann surface, and $\Omega$ is the domain of discontinuity of this free group action. Not only this, but additionally, the image of the $2 g$ boundary circles on the Riemann sphere under the quotient morphism may be identified with $g+g$ oriented loops and their inverses in the fundamental group of $S$.

Note that for a compact Riemann surface of genus $g$, its fundamental group is generated by $2 g$ oriented loops $l_{1}, \ldots, l_{g}$ and $\lambda_{1}, \ldots, \lambda_{g}$ modulo the commutator relations $\prod_{i=1}^{g}\left[l_{i}, \lambda_{i}\right]=1$. Hence, $g$ from the generators of the fundamental group of $S$, for instance $l_{1}$, and $l_{2}$, in the genus-two case, may be identified with the images of the corresponding boundary circles on the Riemann sphere under the quotient morphism $\Omega \rightarrow \Omega / \Theta_{g}$. Moreover, the latter is a covering space projection morphism, such that its group of covering automorphisms constitutes the smallest normal subgroup of the fundamental group of $S$ containing the generators $l_{1}, \ldots, l_{g}$. In this way, the free group on $g$ generators, $\Theta_{g}$, where $g$ is the genus of the Riemann surface, is identified with the quotient of the fundamental group of $S$ modulo the above-defined normal subgroup.

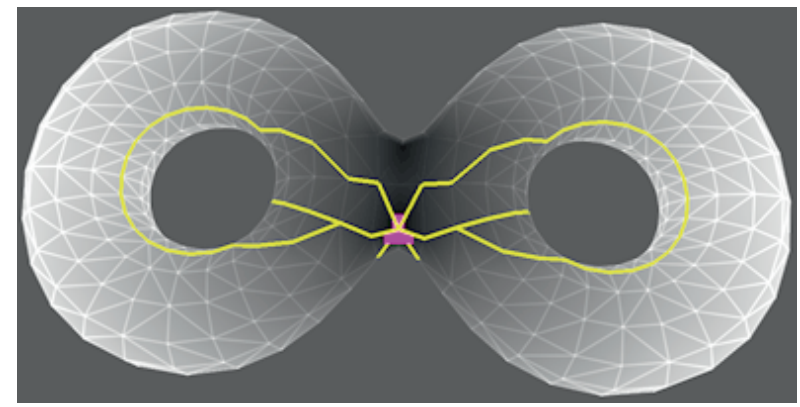

We conclude that while Fuchsian uniformization, utilizes the universal covering space of a Riemann surface with the Fuchsian group corresponding isomorphically to the fundamental group of this Riemann surface, Schottky uniformization, expressed in terms of the action of the 
free non-commutative group, as we have seen, corresponds to the intermediate covering space obtained by means of the quotient of the Fuchsian group by the normal subgroup generated by half of the generators of the fundamental group. Finally, using the fact that $\Theta_{g}$ is a subgroup of $\Theta_{2}$ for every $g>2$, we realize the universal role of the free non-commutative group in two generators $\Theta_{2}$ in the uniformization of Riemann surfaces by means of covering spaces. 

